\title{
Geometric structure and information change in phase transitions
}

\author{
Eun-jin Kim \\ School of Mathematics and Statistics, University of Sheffield, Sheffield S3 7RH, United Kingdom \\ Rainer Hollerbach \\ Department of Applied Mathematics, University of Leeds, Leeds LS2 9JT, United Kingdom
}

(Received 16 February 2017; published 6 June 2017)

\begin{abstract}
We propose a toy model for a cyclic order-disorder transition and introduce a geometric methodology to understand stochastic processes involved in transitions. Specifically, our model consists of a pair of forward and backward processes (FPs and BPs) for the emergence and disappearance of a structure in a stochastic environment. We calculate time-dependent probability density functions (PDFs) and the information length $\mathcal{L}$, which is the total number of different states that a system undergoes during the transition. Time-dependent PDFs during transient relaxation exhibit strikingly different behavior in FPs and BPs. In particular, FPs driven by instability undergo the broadening of the PDF with a large increase in fluctuations before the transition to the ordered state accompanied by narrowing the PDF width. During this stage, we identify an interesting geodesic solution accompanied by the self-regulation between the growth and nonlinear damping where the time scale $\tau$ of information change is constant in time, independent of the strength of the stochastic noise. In comparison, BPs are mainly driven by the macroscopic motion due to the movement of the PDF peak. The total information length $\mathcal{L}$ between initial and final states is much larger in BPs than in FPs, increasing linearly with the deviation $\gamma$ of a control parameter from the critical state in BPs while increasing logarithmically with $\gamma$ in FPs. $\mathcal{L}$ scales as $|\ln D|$ and $D^{-1 / 2}$ in FPs and BPs, respectively, where $D$ measures the strength of the stochastic forcing. These differing scalings with $\gamma$ and $D$ suggest a great utility of $\mathcal{L}$ in capturing different underlying processes, specifically, diffusion vs advection in phase transition by geometry. We discuss physical origins of these scalings and comment on implications of our results for bistable systems undergoing repeated order-disorder transitions (e.g., fitness).
\end{abstract}

DOI: 10.1103/PhysRevE.95.062107

\section{INTRODUCTION}

Phase transition plays a vital role in many disciplines ranging from cosmology, elementary particle theory, condensed matter, chemistry, and biology to social-economic movements [1-5]. In critical phenomena, a set of control parameters such as temperature triggers the transition of a state of matter (e.g., vapor, water, and ice) or magnetization (ferromagnet), leading to the emergence or disappearance of a global mode (macroscopic observable). At a supercritical (disordered) state, the value of an order parameter is zero while at a subcritical (ordered) state, it takes a nonzero value. Order-disorder transition is also at the heart of self-organization [6] whereby coherent structure spontaneously emerges out of complexity, providing a key mechanism for maintaining dynamic balance in nonequilibrium systems. Examples include the formation of shear flows or vortices in fluids or plasmas [7-12] or pattern formation in chemical oscillators, embryogenesis, and even traffic flows. With improved measurement technology in biological experiments, there has been accumulating evidence that similar transitions play a primary role in different biological processes [4]. For instance, Ref. [13] demonstrated an emerging property in whole-genome expression through the transition from a unimodal distribution to a bimodal distribution due to bistability and claimed self-organized criticality (SOC) [14-16].

Bistability, often used as a simple framework to study disorder-order (or supercritical-subcritical), is thought to provide a crucial regulating mechanism in different systems, e.g., in electric circuits [17], in various cellular processes such as cycles, differentiation and apoptosis, regulation of heart, brain, etc. [18-23]. One of the most striking attributes of some bistable systems is continuous switching between ordered and disordered states, the transition occurring in bursts interspersed by a quiescent period (e.g., see [18]). Furthermore, the sandpile model-a prototypical model for SOC [14]—can also be viewed as the repetition of such switching between the buildup of large gradients (forcing) and the sandpile's collapse beyond some critical gradient (dissipation). In fact, recent work by di Santo et al. [24] attempted to formally adapt SOC to bistable systems by invoking self-organized bistability. Our work was motivated to present a different way of understanding disorder-to-order and order-to-disorder transitions in bistable systems, highlighting asymmetry between these two processes.

In this paper, we propose a toy model for a cyclic order-disorder transition, perform a detailed study on the time evolution of probability density functions (PDFs), and introduce a geometric methodology to understand stochastic processes involved in order-disorder transition. Specifically, our model consists of a pair of forward and backward processes for the emergence of a structure and its reverse process, the disappearance of a structure, respectively, in a stochastic environment. We calculate time-dependent PDFs and the total number of different states that a system undergoes during the transition. The latter is quantified by the information length [25-29], which is dimensionless, defined as (see Appendix A)

$$
\mathcal{L}(t)=\int_{0}^{t} \frac{d t_{1}}{\tau\left(t_{1}\right)}=\int_{0}^{t} d t_{1} \sqrt{\int d x \frac{1}{p\left(x, t_{1}\right)}\left[\frac{\partial p\left(x, t_{1}\right)}{\partial t_{1}}\right]^{2}},
$$


where $p(x, t)$ is a time-dependent PDF for a stochastic variable $x$. In Eq. (1), $\tau(t)$ is the time-varying "time unit":

$$
\frac{1}{[\tau(t)]^{2}}=\int d x \frac{1}{p(x, t)}\left[\frac{\partial p(x, t)}{\partial t}\right]^{2} .
$$

$\tau(t)$ in Eq. (2) has dimensions of time, and quantifies the correlation time over which the (dimensionless) information changes, thereby serving as the time unit as far as the information is concerned. The first equality in Eq. (1) represents that $\mathcal{L}$ is the total elapsed time measured in units of $\tau$. Alternatively, the information length represents the total different number of states between the initial and final times, 0 and $t$ respectively, and establishes a distance between the initial and final PDFs in the statistical space. Our information length is based on Fisher information (cf. [30]) and is a generalization of statistical distance [31], where the distance is set by the number of distinguishable states between two PDFs. While the latter was heavily used in equilibrium or near equilibrium of classical and quantum systems [32-40], our recent work [25-29] adapted this concept to a nonequilibrium system to elucidate geometric structure of nonequilibrium processes. Specifically, Ref. [29] mapped out the attractor structure $\mathcal{L}_{\infty}$ vs $x_{0}$ for the linear and cubic process and showed (i) that a linear damping preserves a linear geometry $\mathcal{L}_{\infty} \propto x_{0}$ and (ii) that a nonlinear damping gives rise to a power-law scaling $\mathcal{L}_{\infty} \propto x_{0}^{n}$ ( $n \sim 1.5-1.9)$ of the attractor structure. Reference [28] found interesting geodesic solutions in a nonautonomous OrnsteinUhlenbeck $(\mathrm{OU})$ process by modulating model parameters and by including a time-dependent external deterministic killing term. Notably, the modulation of the model parameters and the killing term were periodic or oscillatory.

Recalling that a geodesic is a particular path minimizing the total information change, it is important to emphasize that it endows a system with the advantage of undergoing the least amount of changes during the nonequilibrium process. This could be extremely beneficial to a system when adjusting to a changing environment takes time and/or is costly. Our previous results summarized above then raise the important question of what basic physical mechanisms are responsible for a geodesic in a more realistic system without tailored time-dependent control. Our previous experience with the cubic process [29] suggests that a nonlinear interaction might be one of them. In this paper, we show that the predator-prey type self-regulation between the positive feedback and the negative feedback in the disorder-to-order transition maintains the system closer to the geodesic, minimizing the information change. That is, we find that the self-regulation with a nonlinear interaction facilitates a geodesic.

Motivated by a quenched experiment like a spinoidal decomposition [3], we induce a sudden change of a control parameter at the initial time of forward and backward processes and study the evolution of an initially far-from-equilibrium unimodal PDF into an equilibrium bimodal PDF during disorder-to-order transition in the forward process, and vice versa in the backward process. From time-dependent PDFs, we calculate the information change associated with a nonequilibrium evolution in forward and backward processes by the information length, highlighting differences. We note that a sudden change in control parameters takes place naturally in self-organized systems, for instance, in gene expressions (e.g., see [18]). We present high-resolution numerical results together with analytical analysis in limiting cases. The remainder of this paper is organized as follows. Section II presents our model. Section III contains analytical results of time-dependent PDFs while Section IV provides numerical solutions. We discuss information length in Sec. V and entropy in Sec. VI. We conclude in Sec. VII. Appendices contain the derivation of equations used in the main text.

\section{MODELS}

We consider the following Langevin equation for a stochastic variable $x$ :

$$
\frac{d x}{d t}=F(x)+\xi=-\lambda(t) x-\mu x^{3}+\xi .
$$

Here,

$$
F(x)=-\lambda x-\mu x^{3}
$$

is a deterministic force. $x$ can represent any order parameter (e.g., velocity, magnetization) and $F(x)$ is a deterministic force, which can be interpreted as the gradient of the potential $U(x)$ as $F(x)=-\frac{\partial U(x)}{\partial x}$. Thus, for FP with $F=\gamma x-\mu x^{3}$, $U=-\frac{\gamma}{2} x^{2}+\frac{\mu}{4} x^{2}$ is a double well potential; for BP with $F=-\gamma x-\mu x^{3}, U=\frac{\gamma}{2} x^{2}+\frac{\mu}{4} x^{2}$ is a monopotential. $\xi$ in Eq. (3) is a white noise with a short correlation time with the following statistical property:

$$
\left\langle\xi(t) \xi\left(t^{\prime}\right)\right\rangle=2 D \delta\left(t-t^{\prime}\right),
$$

where the angular brackets denote the average over $\xi$, and $D$ is the strength of the forcing. With no loss of generality, we fix the value of $\mu(=1)$ and consider $\lambda$ as a control parameter. The Fokker-Planck equation [41,42] corresponding to Eq. (3) is as follows:

$$
\frac{\partial}{\partial t} p(x, t)=\frac{\partial}{\partial x}\left[-F(x)+D \frac{\partial}{\partial x}\right] p(x, t) .
$$

Physically, $\lambda(t)$ can represent the deviation of the temperature from the critical value as $\lambda \propto T-T_{c}$, where $T_{c}$ is the critical temperature: subcritical for $\lambda<0$, supercritical for $\lambda>0$, and critical at $\lambda=0$. For $\lambda<0$, in the absence of the stochastic noise $\xi, x$ has the two equilibrium points $\pm \sqrt{\frac{-\lambda}{\mu}}$, while with $\xi \neq 0$, the equilibrium is described by a bimodal PDF with two peaks at $x= \pm \sqrt{\frac{-\lambda}{\mu}}$. In comparison, for $\lambda>0$, an equilibrium PDF is unimodal with a peak at $x=0$ when $\xi \neq 0$. Equation (3) is motivated by Ginzburg-Landau fields in zero dimension [43] and the extension of our recent work $[29,44]$ on time-dependent PDF and information length for a critical state $\lambda=0$.

In this paper, we consider a time-dependent control parameter $\lambda(t)$ such that in the forward process (FP), $\lambda$ changes from $\lambda=\gamma>0$ to $\lambda=-\gamma<0$, inducing disorder-to-order transition, while in the backward process (BP), $\lambda$ changes from $\lambda=-\gamma<0$ to $\lambda=\gamma>0$, triggering order-to-disorder transition (by definition $\gamma$ is positive here). The FP and BP make up a pair of disorder-to-order and order-to-disorder transitions. A sufficiently slowly changing $\lambda(t)$ would lead to a reversible quasiequilibrium process. In this paper, since we 
TABLE I. Summary of FPs and BPs: $p_{B}$ and $p_{F}$ are equilibrium PDFs of FPs and BPs, respectively.

\begin{tabular}{lcc}
\hline \hline Case & FP & BP \\
\hline$-\lambda$ & $\gamma$ & $-\gamma$ \\
$p(x, 0)$ & $p_{B}(x)$ & $p_{F}(x)$ \\
$p(x, t \rightarrow \infty)$ & $p_{F}(x)$ & $p_{B}(x)$ \\
$\sigma(t=0)$ & $\frac{D}{\gamma}$ & $\frac{D}{2 \gamma}$ \\
$\sigma(t \rightarrow \infty)$ & $\frac{D}{2 \gamma}$ & $\frac{D}{\gamma}$ \\
\hline \hline
\end{tabular}

are interested in a far-from-equilibrium process, we introduce a sudden change $\lambda$ at the beginning of FP and BP as in a quenched experiment. Specifically, we change $\lambda$ from $\lambda=\gamma$ to $\lambda=-\gamma$ at $t=0$ in the FP, and from $\lambda=-\gamma$ to $\lambda=\gamma$ at $t=0$ in the BP, respectively. Alternatively, FPs and BPs are described by a constant $\lambda$ for $t>0$ with initial nonequilibrium PDFs as follows:

(i) Forward process (FP). $\lambda=-\gamma<0$ : at $t=0$, a unimodal PDF with a peak at $x=0$, which evolves into a bimodal PDF with two peaks at $x= \pm \sqrt{\gamma / \mu} \neq 0$ as $t \rightarrow \infty$.

(ii) Backward process (BP). $\lambda=\gamma>0$ : at $t=0$, a bimodal PDF with two peaks at $x= \pm \sqrt{\gamma / \mu} \neq 0$, which evolves into a unimodal PDF with a peak at $x=0$ as $t \rightarrow \infty$.

The FP and the BP have the following equilibrium PDFs $p_{F}(x)$ and $p_{B}(x)$, respectively:

$$
\begin{aligned}
& p_{F}(x) \propto \exp \left[-\frac{\mu}{4 D}\left(x^{2}-\frac{\gamma}{\mu}\right)^{2}\right], \\
& p_{B}(x) \propto \exp \left[-\frac{\mu}{4 D}\left(x^{2}+\frac{\gamma}{\mu}\right)^{2}\right] .
\end{aligned}
$$

It is worth noting that for sufficiently small $D, p_{F}(x)$ and $p_{B}(x)$ can be approximated by Gaussian distributions as (see Appendix B)

$$
\begin{gathered}
p_{F}(x) \sim \frac{\sqrt{\beta_{F}}}{2 \sqrt{\pi}}\left[e^{-\beta_{F}(x+\sqrt{\gamma / \mu})^{2}}+e^{-\beta_{F}(x-\sqrt{\gamma / \mu})^{2}}\right], \\
p_{B}(x) \sim \frac{\sqrt{\beta_{B}}}{\sqrt{\pi}} e^{-\beta_{B} x^{2}},
\end{gathered}
$$

where $\beta_{F}=\frac{\gamma}{D}$ and $\beta_{B}=\frac{\gamma}{2 D}$. Equation (9) represents the sum of the two Gaussians with peaks at $\pm \sqrt{\frac{\gamma}{\mu}}$ and variance $\frac{D}{2 \gamma}$. Our FP and BP allow us to consider an interesting cyclic quenched experiment where we suddenly change $\lambda$ after PDFs settle into equilibrium at the end of the $\mathrm{FP}$ or $\mathrm{BP}$, reset time $t=0$, and then start the BP or FP until they evolve to equilibrium, and potentially repeating indefinitely. A pair of forward or backward processes is thus completed by using $p_{B}(x)$ as an initial condition for FPs and $p_{F}(x)$ for BPs (see Table I). Consequently, the initial PDFs in both FPs and BPs are strongly out of equilibrium, and PDFs undergo transient relaxation. We investigate time-dependent PDFs and information length during this transient relaxation, comparing them in FPs and BPs. In particular, we are interested in how $\mathcal{L}$ depends on $\gamma$ and $D$ (for $\mu=1$ ).

Table I summarizes the value of $\lambda$ in Eq. (3) and initial conditions for FPs and BPs together with the variance $\sigma=$ $\left\langle x^{2}\right\rangle-\langle x\rangle^{2}$, where the angular brackets denote the average over the stochastic noise $\xi$. We note that $p_{F}$ and $p_{B}$ are equilibrium PDFs of FPs and BPs, respectively.

\section{THEORETICAL PREDICTION OF PDFS}

As will be shown later, the time evolution of PDFs in FPs and BPs is significantly different. It is basically because the FP is dominated by the broadening of the PDF due to the stochastic noise and instability in the early stage; $x=0$ is an unstable equilibrium point when $\xi=0$, and the instability slowly builds up due to $\xi$ and a finite width of the initial PDF until $t \propto O(|\ln D|)$ (see later for more details) when the PDF undergoes a considerable change, developing two peaks around $x= \pm \sqrt{\gamma / \mu}$. In comparison, the BP is mainly driven by the movement of the two peaks towards $x=0$ before diffusion becomes crucial in forming a single peak at $x=0$. In this section, we present analytical results before presenting numerical solutions in Sec. IV.

\section{A. Forward process (FP)}

The FP starts with a Gaussian PDF for small $D$ and goes through the following two stages (e.g., see [45-48]): (i) the initial stage of the broadening of the initial Gaussian PDF due to stochastic noise $\xi$ and instability $\gamma$ towards the development of the two peaks at $x= \pm \sqrt{\frac{\gamma}{\mu}}$, and (ii) the final stage (Kramer's regime) of narrowing of the PDF to the final (equilibrium) (double) Gaussian PDF. To understand order formation, it is instructive to examine the evolution of PDF analytically in stage (i) in detail. To this end, it is convenient to transform away the nonlinear term in Eq. (3) into a linear damping term at the expense of a multiplicative-type stochastic noise [49]. We thus look for a variable $y$ such that Eq. (3) becomes $d y / d t=$ $\gamma y+\xi F(y)$ where $F(y)$ is a function of $y$. This is achieved by requiring $d y / d x=\gamma y /\left(\gamma x-\mu x^{3}\right)$, with the solution $x=$ $y / \sqrt{1+\alpha y^{2}}$, where $\alpha=\frac{\mu}{\gamma}$. Specifically, $y$ satisfies

$$
\frac{d y}{d t}=\gamma y+\xi\left(1+\alpha y^{2}\right)^{3 / 2} .
$$

Equation (11) provides a convenient way of computing $y$ during the stage (i) by approximating $\xi\left(1+\alpha y^{2}\right)^{3 / 2} \sim \xi$ for small $y$. Thus, to leading order, $y$ is a Gaussian process, simply given by the Ornstein-Uhlenbeck process [42] with a negative damping. The transition probability of $y$ is therefore given by the following Gaussian PDF:

$$
p\left(y, t ; y_{0}, 0\right)=\sqrt{\frac{\beta_{1}}{\pi}} e^{-\beta_{1}\left(y-y_{0}\right)^{2}},
$$

where $y_{0}=\frac{x_{0}}{\sqrt{1-\alpha x_{0}^{2}}}$ and $\frac{1}{\beta_{1}(t)}=\frac{D}{\gamma}\left(e^{2 \gamma t}-1\right)$. In terms of $y$, the initial $\operatorname{PDF} p(y, 0)$ is given by

$$
p(y, 0)=\sqrt{\frac{\beta_{0}}{\pi}} e^{-\beta_{0}\left[y_{0}^{2} /\left(1+\alpha y_{0}^{2}\right)\right]} \sim \sqrt{\frac{\beta_{0}}{\pi}} e^{-\beta_{0} y_{0}^{2}}
$$

for a narrow initial PDF with $\beta_{0} y_{0}^{2} \ll 1$ (e.g., for small $D$ ). Here, $\beta_{0}=\frac{1}{2\left\langle\left(\delta y_{0}\right)^{2}\right\rangle}$ is the initial inverse temperature due to the 
finite width of $p(x, 0)$. Equations (12) and (13) give us

$$
p(y, t)=\sqrt{\frac{\beta_{0}}{\pi}} \sqrt{\frac{\beta_{1}}{\pi}} \int_{-\infty}^{\infty} d y_{0} e^{-\beta_{1}\left(y-y_{0}\right)^{2}} e^{-\beta_{0} y_{0}^{2}}=\sqrt{\frac{\beta}{\pi}} e^{-\beta y^{2}} .
$$

Here, $\beta=\frac{1}{2\left\langle(\delta y)^{2}\right\rangle}$ is the inverse temperature given by

$$
\frac{1}{\beta(t)}=\frac{1}{\beta_{0}} e^{2 \gamma t}+\frac{2 D}{\gamma}\left(e^{2 \gamma t}-1\right) .
$$

This can alternatively be expressed in terms of the variance $\sigma=\left\langle(\delta y)^{2}\right\rangle=\frac{1}{2 \beta}$ :

$$
\sigma(t)=\frac{1}{2 \beta(t)}=\sigma_{0} e^{2 \gamma t}+\frac{D}{\gamma}\left(e^{2 \gamma t}-1\right),
$$

where $\sigma_{0}=\frac{1}{2 \beta_{0}}=\left\langle\left(\delta y_{0}\right)^{2}\right\rangle=\frac{D}{\gamma}$ is the initial variance. For $t>$ $\frac{1}{2 \gamma}$, Eqs. (15) and (16) become

$$
\beta \sim \frac{\gamma}{D} e^{-2 \gamma t}, \quad \sigma \sim \frac{2 D}{\gamma} e^{2 \gamma t}
$$

We see from this that when $D$ is changed to $D_{1}(<D)$, and simultaneously $t$ is changed to $t_{1}$ according to

$$
t_{1}-t=\frac{1}{2 \gamma} \ln \left[\frac{D}{D_{1}}\right]
$$

the variance remains the same. The Gaussian PDF of $y$ in Eq. (14) is a good approximation in stage (i) before the settlement of the PDF into final equilibrium with two peaks at $x= \pm \sqrt{\frac{\gamma}{\mu}}$ in stage (ii). We now examine $p(x, t)$ corresponding to Eq. (14).

By using the conservation of the probability $p(x, t) d x=$ $p(y, t) d y$ and Eq. (14) $\left[\int d y p(y, t)=1\right]$, we obtain the PDF of $x$ as follows:

$$
p(x, t)=\frac{1}{\left(1-\alpha x^{2}\right)^{\frac{3}{2}}} \sqrt{\frac{\beta}{\pi}} e^{-\beta\left[x^{2} /\left(1-\alpha x^{2}\right)\right]},
$$

which recovers the previous results $[45,46]$ when $\alpha=1$. To understand the evolution of the peak of the PDF, we calculate the location $x_{m}$ where PDF takes its local maximum or minimum from $\left.\frac{\partial}{\partial x} \ln p(x, t)\right|_{x=x_{m}}=0$, which satisfies

$$
\alpha x_{m}^{2}=0, \text { or } 1-\frac{2 \beta}{3 \alpha} \text {. }
$$

Since $\partial_{x x} \ln p(x, t)=3 \alpha-2 \beta$ at $x=0, x=0$ is a local minimum for $2 \beta<3 \alpha$ while it is a local maximum for $2 \beta>3 \alpha$. We can easily show that $x=0$ is a local maximum at $t=0$ since $\beta_{0}=\frac{1}{2 \sigma_{0}}=\frac{\gamma}{2 D} \gg \alpha$ for small $D$ where $\sigma_{0}=\frac{D}{\gamma}$ and $\alpha=\frac{\mu}{\gamma}$. $x=0$ remains as a local maximum until $t=t_{c}$ when

$$
2 \beta\left(t_{c}\right)=\frac{1}{\sigma\left(t_{c}\right)}=3 \alpha .
$$

Solving Eq. (21) with the help of Eq. (16) leads to

$$
t_{c} \sim-\frac{1}{2 \gamma} \ln \left[3 \alpha\left(\sigma_{0}+\frac{D}{\gamma}\right)\right] \sim-\frac{1}{2 \gamma} \ln \left(\frac{6 D \mu}{\gamma^{2}}\right),
$$

where $\sigma_{0}=\frac{D}{\gamma}, \alpha=\frac{\mu}{\gamma}$, and $e^{2 \gamma t}-1 \sim e^{2 \gamma t}$ were used for $t>\frac{1}{2 \gamma} \cdot t_{c}$ in Eq. (22) signifies the development of a plateau at $x=0$ due to the formation of the two peaks at $x \neq 0$ and sets the time scale beyond which Eqs. (14) and (19) cannot reasonably describe the time evolution of the PDFs. Equation (22) demonstrates that the two peaks form at a finite time which increases with $|\ln D|$.

The formation of the two peaks discussed above is accompanied by a large (anomalous) fluctuation. That is, $t_{c}$ in Eq. (22) also represents the time scale for strong fluctuations, as shall be confirmed later. We now compute second and fourth moments of $x$ by using Eq. (14) as follows (see Appendix C):

$$
\left\langle x^{2}\right\rangle=\frac{2}{\alpha}\left[\frac{1}{2}-\sqrt{\frac{\beta}{\alpha}} e^{\frac{\beta}{\alpha}} \operatorname{Erfc}\left(\sqrt{\frac{\beta}{\alpha}}\right)\right],
$$

where $\operatorname{Erfc}(Q)=\int_{Q}^{\infty} d y e^{-y^{2}}=\frac{\sqrt{\pi}}{2} \operatorname{erfc}(Q) ; \quad \operatorname{erfc}(Q)=$ $\frac{2}{\sqrt{\pi}} \int_{Q}^{\infty} d y e^{-y^{2}}$ is the complementary error function. Similarly, the fourth moment is found (see Appendix D) as follows:

$$
\left\langle x^{4}\right\rangle=\frac{2}{\alpha^{2}}\left[\frac{1}{2}-\left(\frac{3}{2}+\frac{\beta}{\alpha}\right) \sqrt{\frac{\beta}{\alpha}} e^{\beta / \alpha} \operatorname{Erfc}\left(\sqrt{\frac{\beta}{\alpha}}\right)+\frac{\beta}{2 \alpha}\right]
$$

In the limit of large $\beta$ corresponding to small time [see Eq. (15)], we can obtain the following approximate expressions for Eqs. (23) and (24):

$$
\left\langle x^{2}\right\rangle \sim \frac{1}{2 \beta},\left\langle x^{4}\right\rangle \sim \frac{3}{4 \beta^{2}},
$$

where we used the asymptotic expression

$$
\operatorname{Erfc}(Q)=\frac{e^{-Q^{2}}}{2 Q}\left[1-\frac{1}{2 Q^{2}}+\frac{3}{8 Q^{3}}-\frac{15}{8 Q^{4}}+\cdots\right]
$$

for large $Q$. It is interesting to note that the value of $\left\langle x^{2}\right\rangle$ and $\left\langle x^{4}\right\rangle$ in Eq. (25) depending only on $\beta$ is due to stochastic noise, with no dependence on the peak position $x= \pm \sqrt{1 / \alpha}$. Furthermore, we observe that $\left\langle x^{2}\right\rangle$ and $\left\langle x^{4}\right\rangle$ are related by $\left\langle x^{4}\right\rangle=3\left\langle x^{2}\right\rangle^{2}$, as is often found in the Gaussian process. This is consistent with our discussion above about stage (i) before the formation of order. In comparison, in the opposite limit of small $\beta \rightarrow 0$ for large time, we find to leading order

$$
\left\langle x^{2}\right\rangle \sim \frac{1}{\alpha}=\frac{\gamma}{\mu},\left\langle x^{4}\right\rangle=\frac{1}{\alpha^{2}}=\left\langle x^{2}\right\rangle^{2},
$$

which are due to the formation of two peaks at $x= \pm \sqrt{1 / \alpha}$. Equations (25) and (27) will be confirmed by numerical simulations in Sec. IV.

Finally, the second and fourth moments in Eqs. (23) and (24) also play a role in determining the energy budget in the systems. To see this, we multiply Eq. (3) by $x$ and take the average over $\xi$ and the initial condition to obtain the following equation:

$$
\frac{1}{2} \frac{d\left\langle x^{2}\right\rangle}{d t}=\gamma\left\langle x^{2}\right\rangle-\mu\left\langle x^{4}\right\rangle+D .
$$

Here, the last term $D$, representing the rate of energy injection by $\xi$, was calculated as $\langle\xi(t) x(t)\rangle=\left\langle\xi(t) \int_{0}^{t} d t_{1}\left[\gamma x\left(t_{1}\right)-\right.\right.$ $\left.\left.\mu x\left(t_{1}\right)^{3}+\xi\left(t_{1}\right)\right]\right\rangle=D$. This will be shown to be an exact result in Sec. IV. The middle term $\gamma\left\langle x^{2}\right\rangle-\mu\left\langle x^{4}\right\rangle \equiv H$ represents 
the energy into the system or environment, depending on the sign. When $H>0$, the energy goes into the system, contributing to the increase in $\left\langle x^{2}\right\rangle$; when $H<0$, the energy is dissipated in the system, increasing heat in the environment. The sign change in $H$ will also be confirmed in Sec. IV. It is interesting to see from Eq. (25) that the crossover between these two cases occurs at time when $2 \beta\left(t_{c}\right)=3 \alpha$, which is the same as Eq. (21). Therefore, for $t<t_{c}$, energy goes into the system $(H>0)$ while for $t>t_{c}$, energy is dissipated in the system, increasing heat in the environment $(H<0)$. Thus, settling into the final equilibrium PDF involves the dissipation with $H<0 . H>0(H<0)$ is related to the increase (decrease) in the differential entropy in Sec. VI. Interestingly, the fluctuation in $H$ is symptomatic of a predatorprey type self-regulating, oscillatory behavior and is related to a geodesic, as discussed in Sec. VI.

\section{B. Backward process (BP)}

The BP starts with the initial PDF which has two peaks at $\pm \sqrt{\gamma / \mu}$, which is the final equilibrium PDF for the FP. For sufficiently small $D$, the two peaks are far away from $x=0$ and the PDF is approximated as the sum of the two Gaussian PDFs given by Eq. (9). The latter evolve almost independently in $x>$ 0 and $x<0$, respectively, until $t \sim O\left(\ln D^{-1}\right)$ when PDFs merge and undergo significant change in the shape with large fluctuation (see Sec. IV). Since the evolution before merging is dominated by Gaussian evolution, the analysis becomes much simpler compared to the FP. To analyze this Gaussian evolution, we can consider mean value and variance in $x>0$ or $x<0$ separately and treat fluctuation as small compared to the mean value. Specifically, we let $x=z+\delta x$ where $z=\langle x\rangle$ is the mean component averaged over $\xi$ and the initial PDF in $x>0$ (or $x<0$ ) while $\delta x$ is the fluctuation $\langle\delta x\rangle=0$. For the $\mathrm{BP}$, we have

$$
\begin{gathered}
\frac{d}{d t} z=-\gamma z-\mu z^{3}-3 \mu\left\langle(\delta x)^{2}\right\rangle z \sim-\gamma z-\mu z^{3}, \\
\frac{d}{d t} \delta x=-\gamma \delta x-3 \mu z^{2} \delta x+\xi
\end{gathered}
$$

where the fluctuation $\left\langle(\delta x)^{2}\right\rangle$ is ignored compared to the mean value $z^{2}$ in Eq. (29). By multiplying Eq. (30) by $\delta x$ and then taking the average over $\xi$ with the help of Eq. (2), we obtain the equation for the variance $\sigma \equiv\left\langle(\delta x)^{2}\right\rangle$ as follows:

$$
\frac{d}{d t} \sigma=-2 \gamma \sigma-6 \mu z^{2} \sigma+2 D
$$

The solutions to Eqs. (29) and (30) are as follows:

$$
\begin{aligned}
{[z(t)]^{2} } & =\frac{z_{0}^{2}}{\left(1+\alpha z_{0}^{2}\right) e^{2 \gamma t}-\alpha z_{0}^{2}}, \\
\sigma(t) & =\frac{\sigma_{0}}{F^{\prime}(t)}+2 D \frac{F(t)}{F^{\prime}(t)},
\end{aligned}
$$

where $F^{\prime}(t)=\left[\left(1+\alpha z_{0}^{2}\right) e^{2 \gamma t}-\alpha z_{0}^{2}\right]^{3} e^{-4 \gamma t}$ and $F(t)=$ $\int_{0}^{t} F^{\prime}\left(t_{1}\right) d t_{1}, z_{0}=\sqrt{\frac{\gamma}{\mu}}, \alpha=\frac{\mu}{v}$, and $\sigma_{0}=\sigma(t=0)=\frac{D}{2 \gamma}$ [see Eq. (9) and Table I)]. The initial evolution of PDFs in the BP is dominated by the movement of the peak given by Eq. (32). As $z$ then decreases exponentially as $z^{2} \sim \frac{1}{2} z_{0}^{2} e^{-2 \gamma t}$ for $t>\frac{1}{2 \gamma}$, the effect of the nonlinear term $\mu z^{2}$ in Eq. (31) is important only for small time $t<\frac{1}{2 \gamma}$. For $t>\frac{1}{2 \gamma}$, we can approximate $F^{\prime}(t)$ and $F(t)$ as

$$
F^{\prime}(t) \sim 8 e^{2 \gamma t}, \quad F(t) \sim \frac{4}{\gamma} e^{2 \gamma t} .
$$

This, together with $\sigma_{0}=\sigma(t=0)=\frac{D}{2 \gamma}$, then simplifies Eq. (33) as

$$
\sigma \sim \frac{D}{\gamma}
$$

Since $z$ in Eq. (32) decreases exponentially as $z^{2} \sim \frac{1}{2} z_{0}^{2} e^{-2 \gamma t}$ for $t>\frac{1}{2 \gamma}, \sigma$ due to diffusion $D$ in Eq. (35) eventually becomes important for the evolution of the PDF, say, at time $t=t_{m}$ when $\sigma$ in Eq. (35) becomes comparable to $z^{2} \sim \frac{1}{2} z_{0}^{2} e^{-2 \gamma t}$ :

$$
t_{m} \sim \frac{1}{2 \gamma} \ln \left[\frac{\gamma z_{0}^{2}}{2 D}\right] .
$$

Because $z$ and $\sigma$ determine the value of different moments (e.g., $\left\langle x^{2}\right\rangle=z^{2}+\sigma$ ), the ratio of different moments (e.g., $\sqrt{\left\langle x^{4}\right\rangle} /\left\langle x^{2}\right\rangle$ ) is essentially set by $t_{m}$ in Eq. (36) and is thus invariant under the simultaneous change of $t$ and $D$ according to Eq. (18), as found in the FP. Also, $t_{m}$ increases with $\ln D^{-1}$, leading to a longer duration of the time interval where the PDF simply moves its position before $D$ becomes important.

Equations (32) and (33) together with $\beta=\frac{1}{2 \sigma}$ determine the evolution of the Gaussian PDF for BP as

$$
p(x, t)=\frac{1}{2} \sqrt{\frac{\beta}{\pi}}\left[e^{-\beta(x-z)^{2}}+e^{-\beta(x+z)^{2}}\right] .
$$

Equations (32) and (33) will also be used for computing $\mathcal{L}$ in Sec. V. Equation (37) becomes invalid when the peaks start merging around $x=0$. The merging happens when $z^{2}$ in Eq. (32) is within the variance $\frac{D}{\gamma}$ in Eq. (35) of the final PDF, roughly around $t \sim t_{m}$ given in Eq. (36).

\section{NUMERICAL SOLUTIONS}

Without loss of generality, any finite interval in $x$ can always be rescaled to $x \in[-1,1]$. If the initial condition is also restricted well away from the boundaries, then solving Eq. (6) on $x \in[-1,1]$ with boundary conditions $p=0$ at $x= \pm 1$ is an excellent match to an unbounded interval. By rescaling $t, \gamma$, and $D$, we can similarly fix $\mu=1$, thereby reducing the number of parameters that need to be varied numerically to only $\gamma$ and $D$. The numerical procedure then involves second-order finite differencing in both space and time, using $O\left(10^{6}\right)$ grid points in $x$, and time steps as small as $O\left(10^{-7}\right)$. Values of $\gamma$ in the interval $[0,0.7]$ were considered. The upper limit ensures that even the double-peak distribution, the final state for the FP and the initial state for the BP, does not encroach on the boundaries $|x|=1$. Values of $D$ from $10^{-3}$ to $10^{-7}$ were considered.

Figure 1 shows a simple but surprisingly useful diagnostic quantity, namely the ratio $\sqrt{\left\langle x^{4}\right\rangle} /\left\langle x^{2}\right\rangle$. For $D \ll 1$ this ratio is observed to be $\sqrt{3}$ for the single peak and 1 for the double peak, consistent with Eqs. (25) and (27), respectively. The FP must therefore yield $\sqrt{3} \rightarrow 1$, and the BP $1 \rightarrow \sqrt{3}$. As seen 


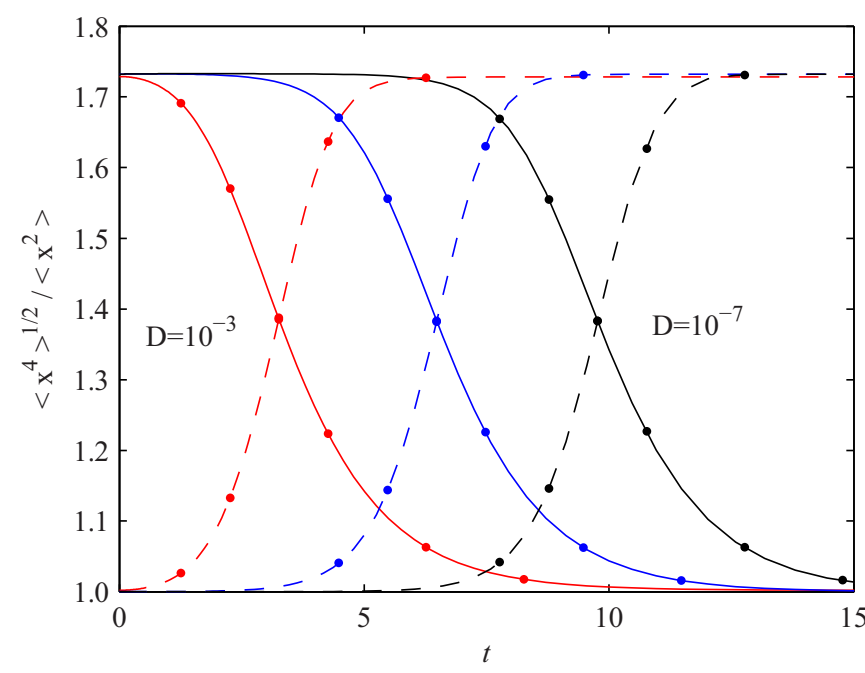

FIG. 1. The ratio $\sqrt{\left\langle x^{4}\right\rangle} /\left\langle x^{2}\right\rangle$ as a function of time, for the three values $D=10^{-3}, 10^{-5}$, and $10^{-7}$, from left to right as indicated. Solid lines denote FP, dashed lines BP. The dots on the lines correspond to the particular snapshots shown in Figs. 2 and 3. The dots on the $D=10^{-3}$ curves (red) are at $t=1.26,2.26,3.26,4.26,6.26$, and 8.26; the dots on the $D=10^{-5}$ curves (blue) are at $t=4.48,5.48$, $6.48,7.48,9.48$, and 11.48; the dots on the $D=10^{-7}$ curves (black) are at $t=7.77,8.77,9.77,10.77,12.77$, and 14.77 . The times 3.26 , 6.48 , and 9.77 are the values where corresponding solid and dashed lines cross; the other times are then related by fixed offsets either before or after these crossing times. Finally, $\gamma=0.7$ for all six curves.

in Fig. 1, the variation with $D$ in this adjustment process is such that every reduction of $D$ by a factor of 100 shifts the curves by a constant amount in time. That is, a time $c \ln D^{-1}$ must elapse before the ratio begins to deviate significantly from its initial value, and start the adjustment process to the other value. The numerically determined value of $c$ is 0.71 , in perfect agreement with the analytic prediction $\frac{1}{2 \gamma}=0.71$ from Eq. (18) for the FP and Eq. (36) for the BP. It is interesting that the two processes not only have the same $c \ln D^{-1}$ time shift, but even the same value of $c$, even though the physical origin of the shifts is so different for the two processes. In Fig. 1, the dots where corresponding solid and dashed lines cross occur at $t=3.26,6.48,9.77$. This crossing time is discussed below in relation to Figs. 2 and 3.

Figure 2 shows the spatial structure of $p(x, t)$ for the FP. The six snapshots correspond to the dots on the curves in Fig. 1. That is, the time shift $c \ln D^{-1}$ according to Eq. (18) is taken into account, and solutions with different values of $D$ are compared at the times when they have the same ratios $\sqrt{\left\langle x^{4}\right\rangle} /\left\langle x^{2}\right\rangle$. We see that the solutions are identical for all three values of $D$, even though the initial conditions obviously depend strongly on $D$. Once the initial diffusive spreading of the central peak has occurred, the subsequent evolution is dominated by the broadening of the PDFs due to the instability $(\gamma)$, and is thus independent of $D$. It is only at the end of the process, when the final double-peak solution is emerging, that $D$ reasserts itself and again determines the width of the peaks.

Figure 3 shows $p(x, t)$ for the $\mathrm{BP}$, again at the times indicated by the dots in Fig. 1. [The evolution before these times is not shown, but was verified to be a motion of the peaks toward the origin in accordance with Eq. (32).] At these appropriately shifted times [e.g., according to Eq. (36)], the solutions are again identical and independent of $D$, provided that a rescaling of both $x$ and $p$ is now also accounted for, with $x$ scaling as $D^{1 / 2}$, and $p$ as $D^{-1 / 2}$. This scaling relation stems from the Gaussian evolution in Eq. (37) where $\beta \propto D^{-1}$. Note that the thick curves in Figs. 2 and 3 correspond to the times when the FP and the BP have the same values of $\sqrt{\left\langle x^{4}\right\rangle} /\left\langle x^{2}\right\rangle$ according to Fig. 1, the solid curves for the FP and dashed curves for the BP crossing each other. On the other hand, the PDFs in the thick curves in Fig. 2 are about to develop two peaks, suggesting that this crossing happens around the theoretically predicted time $t_{c}$ in Eq. (22). By inserting numerical values of $\gamma, \mu=1$, etc., in Eq. (22), we obtain $t_{c}=3.1,6.4,9.8$ for $D=10^{-3}, 10^{-5}$, and $10^{-7}$, respectively. These values are amazingly close to the numerically determined values $t=3.26,6.48,9.77$ from Fig. 1, discussed above. Furthermore, the PDFs in the thick
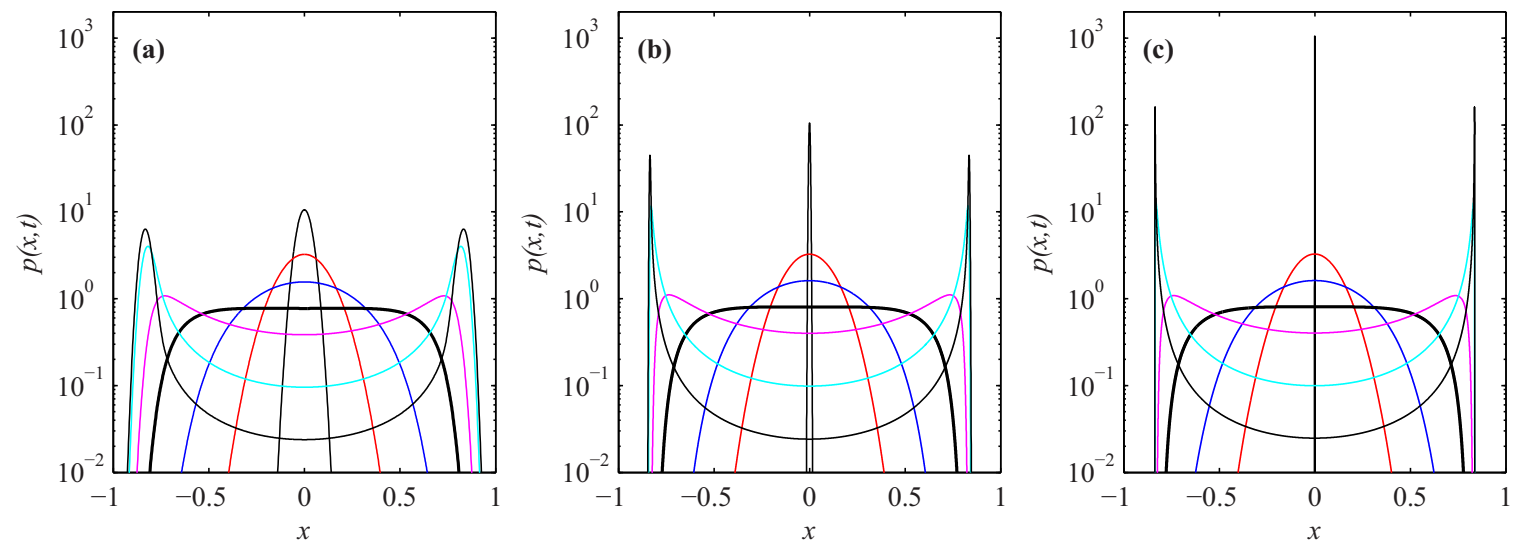

FIG. 2. The PDFs for FP, for (a) $D=10^{-3}$, (b) $D=10^{-5}$, (c) $D=10^{-7}$. The initial condition is given by the central peak. At later times this central value $p(0, t)$ monotonically decreases. The particular times shown are as indicated by the dots in Fig. 1. The thick (black) lines where the central region is flattest correspond to the crossing points in Fig. 1, at times 3.26 in (a), 6.48 in (b), 9.77 in (c); the times for other lines are offset relative to these values as in Fig. 1. Note how the curves at intermediate times are independent of $D$, once this $c \ln D^{-1}$ shift in time is taken into account. 

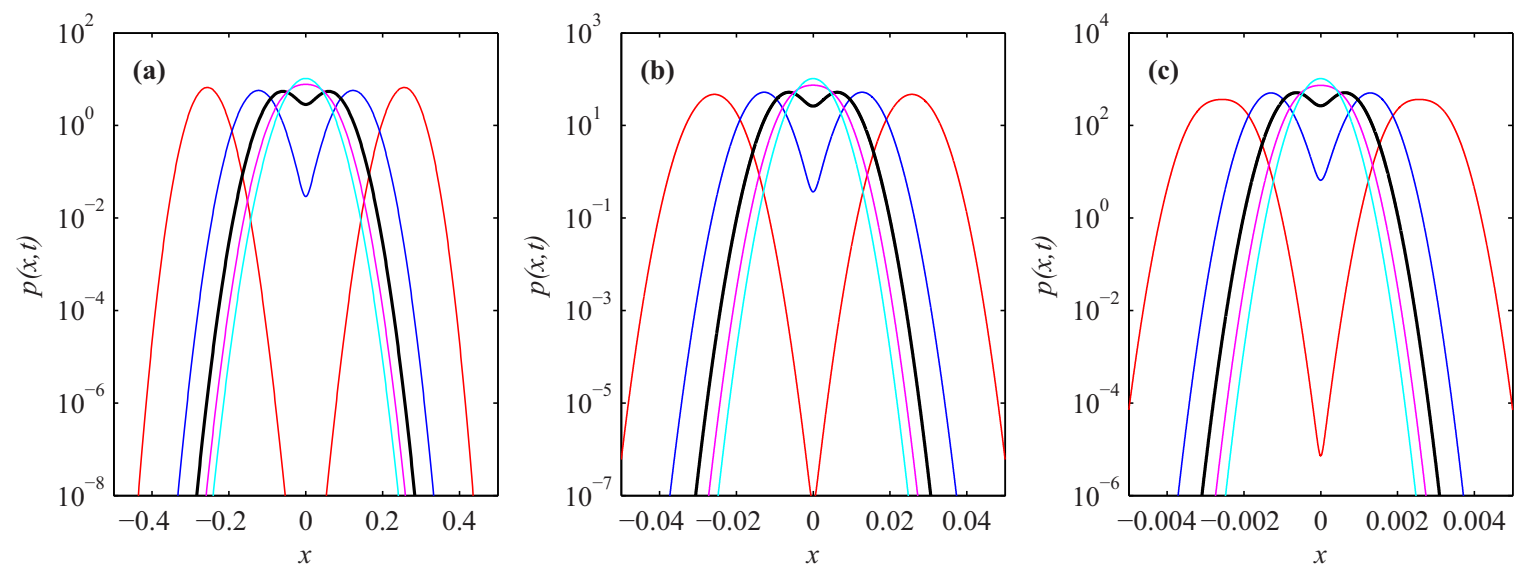

FIG. 3. The PDFs for BP, for (a) $D=10^{-3}$, (b) $D=10^{-5}$, (c) $D=10^{-7}$, at the times indicated in Fig. 1. At the earliest time shown there are still two distinct peaks, which subsequently merge into one single peak. As in Fig. 2, the thick (black) lines where the central region is relatively flat correspond to the crossing points in Fig. 1, at times 3.26 in (a), 6.48 in (b), 9.77 in (c). The times for other lines are again offset relative to these values as in Fig. 1. Note how the curves are independent of $D$, once the shift in time is taken into account, as well as the rescaling of $x$ and $p$.

curves in Fig. 3 suggest that $t_{c}$ is also close to the merging time $t_{m}$ in Eq. (36). We confirm this by inserting in the parameter values in Eq. (36) as $t_{m}=3.9,7.2,10.8$ for $D=10^{-3}, 10^{-5}$, and $10^{-7}$, which are quite close to $t_{c}$.

Figure 4 shows the various terms that make up the energy balance Eq. (28) for FP and its equivalent with $\gamma \rightarrow-\gamma$ for the BP. For the FP $\left\langle x^{2}\right\rangle$ is monotonically increasing, hence $\frac{d}{d t}\left(\left\langle x^{2}\right\rangle / 2\right)$ is always positive. $\frac{d}{d t}\left(\left\langle x^{2}\right\rangle / 2\right)$ initially increases, driven by $\gamma\left\langle x^{2}\right\rangle$, before eventually decreasing again once $\gamma\left\langle x^{2}\right\rangle$ is balanced by $-\left\langle x^{4}\right\rangle$. We observe the change of the sign $H=\gamma\left\langle x^{2}\right\rangle-\left\langle x^{4}\right\rangle$ at $t \sim t_{c}$ in Eq. (22). Unlike $\left\langle x^{2}\right\rangle$ and $\left\langle x^{4}\right\rangle$ which monotonically increase in time, the difference $\Delta=\sqrt{\left\langle x^{4}\right\rangle}-\left\langle x^{2}\right\rangle$ is not monotonic, but increases to its maximum before decreasing to zero as the PDF settles into its equilibrium (figure not shown). This large fluctuation $\Delta$ signifies the phase transition from disordered to ordered states due to the development of the two peaks, which occurs on time scale $t_{c}$ in Eq. (22) which increases with $\ln D^{-1}$.

It is interesting to see from Fig. 4 that the work done by the random forcing, which is the residual $\frac{d}{d t}\left(\left\langle x^{2}\right\rangle / 2\right)-$ $\gamma\left\langle x^{2}\right\rangle+\left\langle x^{4}\right\rangle=\langle\xi(t) x(t)\rangle$, takes the value of $D$ for all time, in agreement with the prediction Eq. (28). This confirms that the nonlinear term does not affect the energy injection from the random forcing, on the basis of which our solutions in Sec. III A were obtained. For BP, where both $-\gamma\left\langle x^{2}\right\rangle$ and $-\left\langle x^{4}\right\rangle$ are driving $\left\langle x^{2}\right\rangle$ toward zero, the balances are
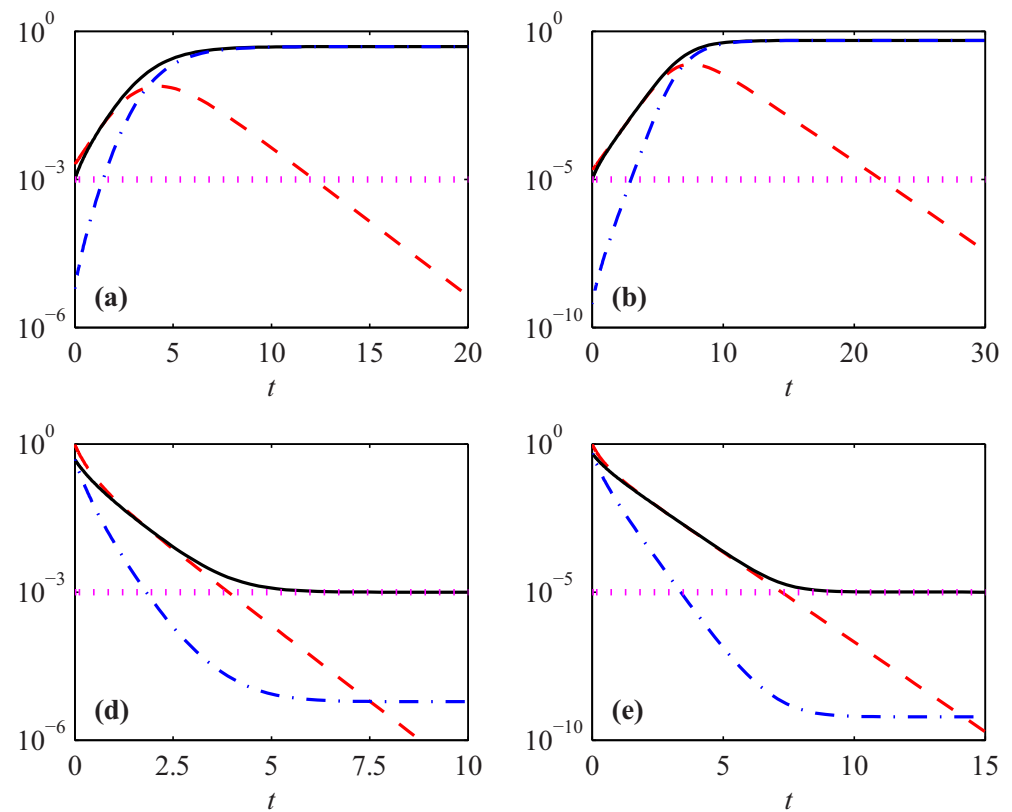
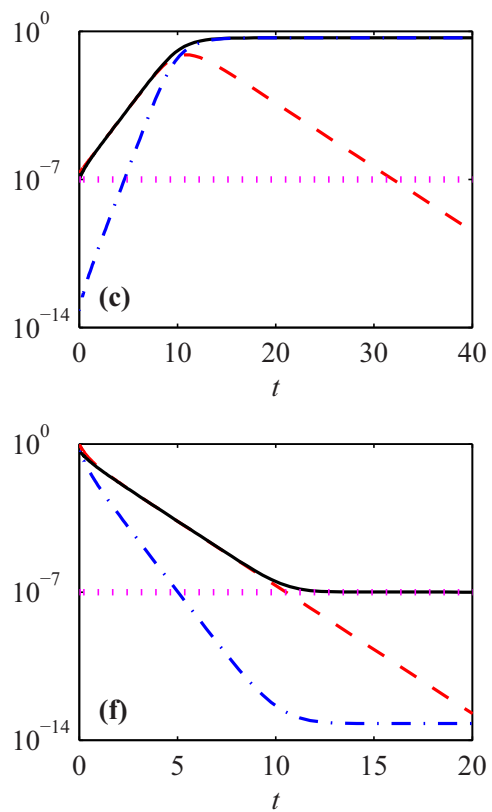

FIG. 4. The first row is for FP, with (a) $D=10^{-3}$, (b) $D=10^{-5}$, (c) $D=10^{-7}$; the second row is for BP, with (d) $D=10^{-3}$, (e) $D=10^{-5}$, (f) $D=10^{-7}$. Dashed curves show $\left|\frac{d}{d t}\left(\left\langle x^{2}\right\rangle / 2\right)\right|$, solid curves $\gamma\left\langle x^{2}\right\rangle$, and dash-dotted curves $\left\langle x^{4}\right\rangle$. The dotted lines show the residuals $\frac{d}{d t}\left(\left\langle x^{2}\right\rangle / 2\right) \mp \gamma\left\langle x^{2}\right\rangle+\left\langle x^{4}\right\rangle$, with - for FP and + for BP. Notice how the residuals are always exactly $D$. 
much simpler. After a very short initial phase where both damping terms are active, and $\frac{d}{d t}\left(\left\langle x^{2}\right\rangle / 2\right)$ decreases faster than exponential, $-\gamma\left\langle x^{2}\right\rangle$ dominates and $\frac{d}{d t}\left(\left\langle x^{2}\right\rangle / 2\right)$ decreases exponentially for all later times. The residual $\frac{d}{d t}\left(\left\langle x^{2}\right\rangle / 2\right)+$ $\gamma\left\langle x^{2}\right\rangle+\left\langle x^{4}\right\rangle=\langle\xi(t) x(t)\rangle$ is again exactly $D$. Finally, we note that settling to the final equilibrium takes less time in BPs than in FPs. For example, if we focus on when the dashed curve drops below the dotted line denoting the residual, that is, when $\left|\frac{d}{d t}\left(\left\langle x^{2}\right\rangle / 2\right)\right|<D$, the time for this to occur in BPs is consistently about $1 / 3$ of that in FPs.

\section{INFORMATION LENGTH}

Having discussed the basic dynamics of the PDFs themselves, we now elucidate the geometric structure and information change through quantities $\mathcal{E}$ and $\mathcal{L}$.

\section{A. Forward process}

For the FP, we recall the excitation of large fluctuations associated with the development of two peaks of a bimodal PDF around $t=t_{c}$ in Eq. (22). Since the change in $\mathcal{L}$ becomes very small for large fluctuation (i.e., large variance of a PDF) around $t=t_{c}$, a good estimate on the total $\mathcal{L}$ can be obtained by considering the change in $\mathcal{L}$ for (i) $t<t_{c}$ during which our results (11)-(19) are valid with Gaussian $p(y, t)$ in Eq. (14) and for (ii) $t>t_{c}$ when $p(x, t)$ is approximated by a Gaussian PDF with narrowing width [45]. We examine these results in detail in the following. First, for $t<t_{c}$, it is advantageous to compute $\mathcal{L}$ by utilizing the Gaussian property of $p(y, t)$ and the invariance of $\mathcal{L}$ under the change of stochastic variables, say, $x$ to $y$, as follows:

$$
\begin{aligned}
\mathcal{L}(t) & =\int_{0}^{t} d t_{1} \sqrt{\int d x \frac{1}{p\left(x, t_{1}\right)}\left[\frac{\partial p\left(x, t_{1}\right)}{\partial t_{1}}\right]^{2}} \\
& =\int_{0}^{t} d t_{1} \sqrt{\int d y \frac{1}{p\left(y, t_{1}\right)}\left[\frac{\partial p\left(y, t_{1}\right)}{\partial t_{1}}\right]^{2}}=\int_{0}^{t} d t \frac{1}{\tau(t)} .
\end{aligned}
$$

Here, we recall that $y=x / \sqrt{\left(1-\alpha x^{2}\right)}\left(x=y / \sqrt{1+\alpha y^{2}}\right)$. For the Gaussian PDFs with mean value $z$ and variance $\sigma, \tau$ in Eq. (2) satisfies

$$
\begin{aligned}
\mathcal{E}=\frac{1}{[\tau(t)]^{2}} & =\frac{1}{2 \beta(t)^{2}}\left(\frac{d \beta}{d t}\right)^{2}+2 \beta\left(\frac{d z}{d t}\right)^{2} \\
& =\frac{1}{2 \sigma(t)^{2}}\left(\frac{d \sigma}{d t}\right)^{2}+\frac{1}{\sigma}\left(\frac{d z}{d t}\right)^{2}
\end{aligned}
$$

Here, $z=\langle y\rangle$ and $\sigma=\left\langle(\delta y)^{2}\right\rangle=1 / 2 \beta$. Since for $t<t_{c},\left\langle x^{2}\right\rangle$ is generated by a stochastic noise [see Eq. (25)] with no mean value $\langle x\rangle=\langle y\rangle=0$, we compute the information length in Eq. (38) by using $p(y, t)$ with the mean value $\langle y\rangle=z \sim 0$. Thus, for $t \leqslant t_{c}$ for the FP, Eqs. (16) and (39) with $z=0$ give us

$$
\mathcal{E} \sim 2 \gamma^{2}\left[\frac{\left(\sigma_{0}+\frac{D}{\gamma}\right) e^{2 \gamma t}}{\sigma_{0} e^{2 \gamma t}+\frac{D}{\gamma}\left(e^{2 \gamma t}-1\right)}\right]^{2} .
$$

By using $\sigma_{0}=\sigma(t=0)=\frac{D}{\gamma}$, we obtain for small $t<\frac{1}{2 \gamma}$

$$
\mathcal{E} \sim 8 \gamma^{2} \frac{1}{[1+2 \gamma t]^{2}},
$$

while in the opposite limit $\frac{1}{2 \gamma}<t<t_{c}$,

$$
\mathcal{E} \sim 2 \gamma^{2}
$$

The characteristic time of the information change $\tau=\frac{1}{\sqrt{\mathcal{E}}}$ follows from Eqs. (41) and (42).

Interestingly, the constant value of $\mathcal{E}$ in Eq. (42) indicates that during this time interval, the PDF follows a geodesic. This geodesic has the characteristic time $\tau=\frac{1}{\sqrt{\mathcal{E}}}=\frac{1}{\sqrt{2} \gamma}$, reflecting that the information flow is due to the instability $\gamma$. As $t_{c} \propto$ $\ln D^{-1}$, the geodesic solution persists for a longer time span for smaller $D$. We confirm these results in Fig. 5 which shows $\mathcal{E}(t), \mathcal{L}(t)$ and $\mathcal{L}_{\infty}(\gamma)$. From Fig. 5(a) we see first that at $t=0$, $\mathcal{E}=3.92=8 \gamma^{2}$ for all $D$. Furthermore, we observe a long plateau where $\mathcal{E}$ is surprisingly constant, with the value $\mathcal{E}=$ $2 \gamma^{2}=0.98$, in perfect agreement with Eq. (42). We recall that this plateau region corresponds precisely to the intermediate stages in Fig. 1, where the ratio $\sqrt{\left\langle x^{4}\right\rangle} /\left\langle x^{2}\right\rangle$ is significantly different from both its initial and final values. We also see in Fig. 5(a) that $\mathcal{E}$ is completely independent of $D$ up until the very final settling in to the double-peak solution, when it decreases exponentially. That $\mathcal{E}$ should be independent of $D$ for most of the process is consistent with our discussion in Secs. III and IV, since we already saw in Fig. 2 that the entire evolution of $p$ is independent of $D$. The initial diffusive broadening of the peak, which does depend on $D$, does not yield a $D$ dependent contribution to $\mathcal{E}$, agreeing with the prediction in Eq. (41).

To calculate $\mathcal{L}$, for $t<t_{c}$, we use Eqs. (38) and (39) with $z=0$ and obtain

$$
\begin{aligned}
\mathcal{L}\left(t_{c}\right) & \sim \frac{1}{\sqrt{2}} \ln \left(\frac{\sigma\left(t_{c}\right)}{\sigma_{0}}\right) \sim \frac{1}{\sqrt{2}} \ln \left[\frac{1}{3 \alpha \sigma_{0}}\right] \\
& \sim \frac{1}{\sqrt{2}}[2 \ln (\gamma)-\ln (3 \mu)-\ln (D)] .
\end{aligned}
$$

On the other hand, during the final stage of PDF evolution in FP for $t>t_{c}$, the location of the two peaks does not change while the width of the PDF changes much more significantly. Thus, we can obtain a useful estimate on $\mathcal{L}$ by taking into account the change in the variance. Furthermore, since $x$ can be approximated as a Gaussian process during this stage [45], we compute total change in $\mathcal{L}$ between $t_{c}$ and $t \rightarrow \infty$ by using the result [Eq. (E9)] for the double Gaussian (see Appendix E):

$$
\begin{aligned}
\mathcal{L}_{\infty}-\mathcal{L}\left(t_{c}\right) & \sim \frac{1}{\sqrt{2}}\left|\ln \left(\frac{\sigma_{F}}{\sigma\left(t_{c}\right)}\right)\right| \sim \frac{1}{\sqrt{2}}\left|\ln \left[3 \alpha \sigma_{F}\right]\right| \\
& \sim \frac{1}{\sqrt{2}}[2 \ln (\gamma)-\ln (2 \mu / 3)-\ln D] .
\end{aligned}
$$

Here, $\sigma_{F}=\sigma(t \rightarrow \infty)=\frac{D}{2 \gamma}$ and $\mathcal{L}_{\infty}=\mathcal{L}(t \rightarrow \infty)$. By adding Eqs. (43) and (44), we obtain the total $\mathcal{L}$ between the initial state at $t=0$ and the final equilibrium state 

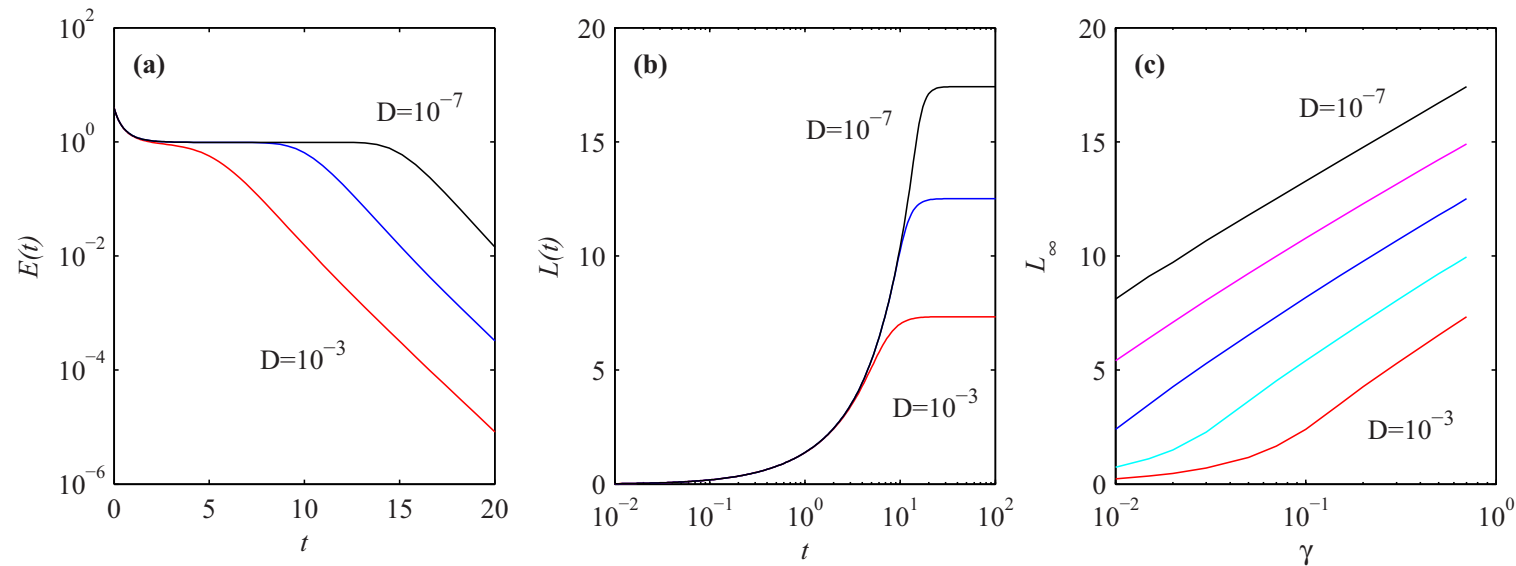

FIG. 5. Panels (a) and (b) show $\mathcal{E}$ and $\mathcal{L}$, respectively, as functions of time, for $\gamma=0.7$. Panel (c) shows $\mathcal{L}_{\infty}$ as a function of $\gamma$. All three panels are for FP only. $D=10^{-3}$ to $10^{-7}$ as indicated. Note the different combinations of linear and logarithmic scales to emphasize different features in different quantities.

as $t \rightarrow \infty$ :

$$
\mathcal{L}_{\infty} \sim \frac{1}{\sqrt{2}}[4 \ln (\gamma)-\ln (2 \mu)-2 \ln D] .
$$

Equation (45) explicitly shows the logarithmic dependence of $\mathcal{L}$ on $\gamma$ and $D$, as observed also in Fig. 5(c). Interestingly, we can now compare Eq. (45) with the following formula that is extracted from Fig. 5(c):

$$
\mathcal{L}_{\infty} \approx 0.5+2.2 \ln \gamma-1.1 \ln D \text {. }
$$

The coefficients of $\ln \gamma$ and $\ln D$ in Eqs. (45) and (46) are in reasonable agreement with each other. The constant term is somewhat different, but the best fit to this term is also very strongly affected by the best fit to the $\ln D$ term, since, e.g., $\left|\ln 10^{-7}\right|=16$, which is already as large as the largest $\mathcal{L}_{\infty}$ in Fig. 5(c). The logarithmic dependence of $\mathcal{L}$ on $\gamma$ and $D$ stems from the diffusive nature of FPs with significant change in the PDF width (large fluctuations).

Note also that for relatively large $D$ and small $\gamma$ the curves in Fig. 5(c) clearly deviate from the otherwise remarkably straight lines. This can be understood by remembering that the widths of the peaks (both single and double) scale as $D^{1 / 2}$, whereas the location of the double peaks is at $x= \pm \sqrt{\gamma}$. If $D \ll \gamma$ were not satisfied, the "initial" and "final" states would therefore already overlap so much that the entire problem becomes uninteresting. For any $\gamma>0$ though, it is always possible to choose $D$ small enough so that the two states are clearly distinct, and the dynamics that lead to Eq. (45) apply.

\section{B. Backward process}

For the BP, both $\mathcal{E}$ and $\mathcal{L}$ behave very differently. From Fig. 6(a), we see that the initial movement of the peaks toward the origin immediately yields a very strongly $D$ dependent $\mathcal{E}$, since narrower peaks yield correspondingly more distinguishable states along the way (e.g., [27,28]). This is responsible for the nonexistence of a geodesic solution with constant $\mathcal{E}$. As indicated in Fig. 6(b), this initial contribution to $\mathcal{L}$ then simply persists throughout the rest of the evolution, with the result that now $\mathcal{L}_{\infty} \propto D^{-1 / 2}$, so each reduction of $D$ by a factor of 100 multiplies $\mathcal{L}_{\infty}$ by 10 . $\mathcal{L}_{\infty}$ for the FP and the BP therefore scale completely differently with $D$, and for sufficiently small $D$ it would be arbitrarily much larger
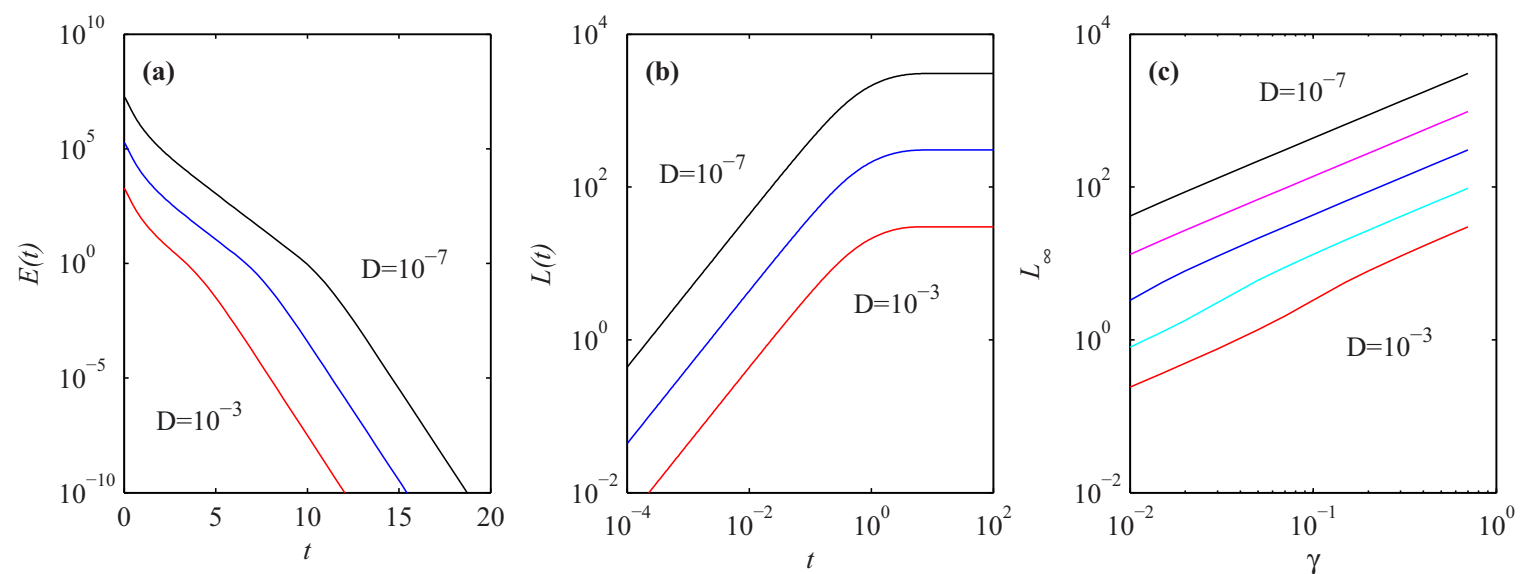

FIG. 6. Panels (a) and (b) show $\mathcal{E}$ and $\mathcal{L}$, respectively, as functions of time, for $\gamma=0.7$. Panel (c) shows $\mathcal{L}_{\infty}$ as a function of $\gamma$. All three panels are for BP only. $D=10^{-3}$ to $10^{-7}$ as indicated. Note the different combinations of linear and logarithmic scales to emphasize different features in different quantities. 
for backward than forward. The variation with $\gamma$ is shown in Fig. 6(c), and yields $\mathcal{L}_{\infty} \approx 1.4 \gamma D^{-1 / 2}$.

We now estimate $\mathcal{L}$ for the BP by taking advantage of the fact that the main contribution to $\mathcal{L}$ comes from the movement of the two peaks and by using Eqs. (32) and (33):

$$
\begin{aligned}
\mathcal{L}(t) & \sim \int_{0}^{t} d t \frac{1}{\sigma} \frac{d z}{d t} \\
& =c_{1}\left(z_{0}\right) \int_{0}^{t} \frac{d t}{\sqrt{\sigma_{0}+2 D F}},
\end{aligned}
$$

where $\quad c_{1}\left(z_{0}\right)=\gamma z_{0}+\mu z_{0}^{3}=2 \gamma z_{0}=2 \gamma \sqrt{\frac{\gamma}{\mu}} \quad\left(z_{0}=\sqrt{\frac{\gamma}{\mu}}\right)$. From Eq. (47), we obtain the lower bound on $\mathcal{L}$ in the long time limit,

$$
\mathcal{L}_{\infty} \sim 2 \gamma z_{0} \int_{0}^{1 / 2 \gamma} \frac{d t}{\sqrt{\sigma_{0}+2 D t}} \sim(\sqrt{6}-\sqrt{2}) \frac{\gamma}{\sqrt{\mu D}},
$$

where $\sigma_{0}=\sigma(t=0)=\frac{D}{2 \gamma}$ for the BP (see Table I) was used. Equation (48) explicitly shows the linear dependence of $\mathcal{L}$ on $\gamma$, as observed in Fig. 6(c). Furthermore, the coefficient of 1.4 in the numerical formula agrees well with the analytic result $(\sqrt{6}-\sqrt{2})=1.0$, which is expected to be an underestimate. The origin of the scaling of $\mathcal{L}_{\infty}$ with $D^{-1 / 2}$ can be traced to the fact that the width of the PDF $\left(\propto D^{-1 / 2}\right)$ does not change much in the BP and a statistically different state is encountered whenever the peak moves the distance of the PDF width $\propto$ $D^{1 / 2}$. A similar $\mathcal{L}_{\infty} \propto D^{-1 / 2}$ was also found in both linear and cubic processes in [29]. The scaling $\mathcal{L} \propto \gamma$ essentially comes from measuring the location of the initial PDF $\propto \gamma^{1 / 2}$ in units of the PDF width $\propto \gamma^{-1 / 2}$. Note that the contribution from $t>\frac{1}{2 \gamma}$ would give scalings that are a bit more complex, as shown in Fig. 6.

\section{ENTROPY}

To complement our analysis above using $\mathcal{L}$, we now look at how the (Gibbs) differential entropy $S(t)=$ $-\int d x p(x, t) \ln p(x, t)$ (e.g., see [50], and using units where the Boltzmann constant $\left.K_{B}=1\right)$ changes during the phase transition. It is important to note that $S$ differs from $\mathcal{L}$ in that it only depends on $p$ at any instant in time, but not on the evolution that led to that PDF. Also, we note that the differential entropy is a global measure of complexity, independent of the rearrangement of constituent elements, and can be negative. We first compute analytically $S(t)$ for the equilibrium PDFs of the FP and the BP, $p_{F}$ and $p_{B}$ in Eqs. (7) and (8), respectively, and quantify the difference between the entropy $S_{F}$ and $S_{B}$ for $p_{F}$ and $p_{B}$, respectively. To compute $S_{F}=-\int_{-\infty}^{\infty} d x p_{F}(x, t) \ln p_{F}(x, t)$, we express Eq. (10) as

$$
p_{F}=\frac{\sqrt{\beta_{F}}}{\sqrt{\pi}} e^{-\beta_{F}\left(x^{2}+x_{0}^{2}\right)} \cosh \left(2 \beta_{F} x_{1} x_{0}\right),
$$

where $\beta_{F}=\gamma / D$. Then, we can show that the entropy $S_{F}$ takes the following form [50]:

$$
\begin{aligned}
S_{F}= & \frac{1}{2}\left[1+\ln \frac{\pi}{\beta_{F}}\right]+2 \beta_{F} x_{0}^{2}\left[1-\operatorname{erf}\left(\sqrt{\beta_{F}} x_{0}\right)\right] \\
& -\sqrt{\frac{\beta_{F}}{\pi}} 2 x_{0} e^{-\beta_{F} x_{0}^{2}}+\Delta .
\end{aligned}
$$

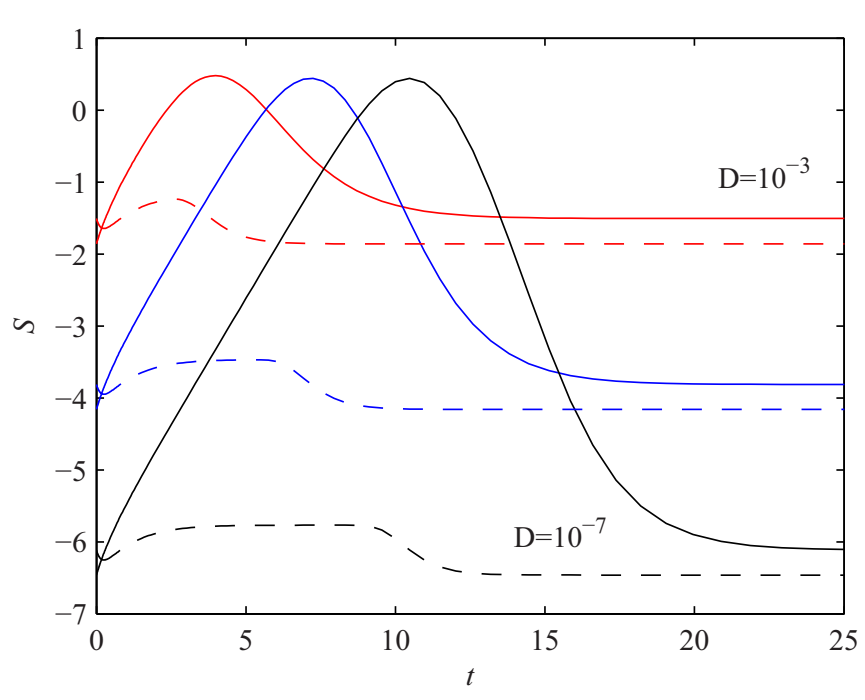

FIG. 7. The entropy $S$ as a function of time, for the three values $D=10^{-3}, 10^{-5}$, and $10^{-7}$, as indicated. Solid lines denote FP, dashed lines BP.

Here, $\operatorname{erf}(x)=\frac{2}{\sqrt{\pi}} \int_{0}^{x} d u e^{-u^{2}}$ is the error function; $\Delta$ is a function of $\beta_{F}$ and $x_{0}$, taking the value $0 \leqslant \Delta \leqslant \ln 2$. For a sufficiently narrow PDF with $\beta_{F} x_{0}^{2} \gg 1, \Delta$ was shown to take a maximum value $\ln 2$ [50]. Since in this limit $\beta_{F} x_{0}^{2} \gg 1$, $\operatorname{erf}\left(\sqrt{\beta_{F}} x_{0}\right) \rightarrow 1$, Eq. (50) is simplified as

$$
S_{F} \sim \frac{1}{2}\left[1+\ln \frac{\pi}{\beta_{F}}\right]+\ln 2=\frac{1}{2}\left[1+\ln \frac{\pi D}{\gamma}\right]+\ln 2 .
$$

For small value of $D$ as used in our numerical simulation, $S_{F}$ is negative, signifying a strongly localized PDF.

For the BF, for simplicity, we use the equilibrium $p_{B}$ in Eq. (10) and $\beta_{B}=\frac{\gamma}{2 D}$ to obtain the corresponding entropy $S_{B}$ as follows:

$$
S_{B}=-\int_{-\infty}^{\infty} d x p_{B} \ln p_{B}=\frac{1}{2}\left[1+\ln \frac{2 \pi D}{\gamma}\right] .
$$

Thus, $S_{F}$ in Eq. (51) is larger than $S_{B}$ by $\frac{1}{2} \ln 2$ due to the formation of the two peaks (see Appendix $\mathrm{F}$ for further discussions).

Figure 7 shows the time evolution of the differential entropy for the FP and the BP. By comparing their values at equilibrium, we see that $S_{F}$ is larger than $S_{B}$ by $\frac{1}{2} \ln 2$, as shown above. That is, the FP and the BP involve a net entropy change $\pm \frac{1}{2} \ln 2$ from one to the other. This overall change in $S$ between the FP and the BP is quite small. However, Fig. 7 shows drastically different time evolution of $S$ in the FP and the BP; a large increase in their value is observed around the phase transition in both processes. Also, Fig. 7 shows clearly that $S$ behaves very differently from $\mathcal{L}$, varying most for the FP, and relatively little for the BP. These results can be understood by recalling from Figs. 2 and 3 that for any given $D, p$ changes its width enormously during the forward evolution, but relatively little going backward. This is reflected in $S$, where all three forward runs peak at the same large values of $S$, when the PDF is broadest, whereas the backward runs have far less variation throughout their entire evolution. The large increase in $S$ in the FP effectively reduces the number of statistically 
different states that a system undergoes in the FP, and thus makes the total information length much smaller compared to the BP. Alternatively, this result shows the emergence of order from disorder is possible through a large entropy increase (also manifested by large fluctuations) in a system. The emergence of order proceeding through a high degree of disorder might be opposite to our naive expectation that the emergence of order should be an outcome of some sort of "ordered act."

The comparison of the FP in Figs. 7 and 5 reveals a very interesting link between the appearance of a geodesic solution with the constant value of $\mathcal{E}$ and the large increase in $S(t)$ followed by its decrease, which is referred to as the entropy oscillation. This behavior is reminiscent of a geodesic solution found in a nonautonomous OU process with an oscillatory time-dependent killing term (or growth rate) together with an oscillatory time-dependent amplitude of the stochastic forcing. Note that the OU process has a linear deterministic force and does not support a geodesic solution without a time-dependent modulation of the parameters. In sharp contrast, the linear growth rate (positive feedback) and nonlinear damping (negative feedback) in the FP in our model is able to sustain a geodesic through the predator-prey type self-regulation - the subtle energy balance between $\gamma\left\langle x^{2}\right\rangle$ and $\left\langle x^{4}\right\rangle$; the large fluctuation in $H=\gamma\left\langle x^{2}\right\rangle-\left\langle x^{4}\right\rangle$ is related to the entropy oscillation and thus the appearance of a geodesic.

\section{CONCLUSION}

We investigated geometric structure during order-todisorder and disorder-to-order transitions in a zerodimensional (0D) Ginzburg-Landau model where the formation (disappearance) of order is modelled by the transition from a unimodal (bimodal) to bimodal (unimodal) PDF of a stochastic variable $x$. We considered off-critical quenching with a pair of forward and backward processes (FPs and BPs) for disorder-to-order and order-to-disorder transition, respectively, by choosing the initial PDF of FPs or BPs the same as the final equilibrium PDF of BPs or FPs. We demonstrated a strikingly different evolution of time-dependent PDFs during transient relaxation due to nonequilibrium initial PDFs in FPs and BPs. In particular, the FP driven by instability undergoes the broadening of the PDF with a large increase in (anomalous) fluctuations before the transition to the ordered state accompanied by narrowing the PDF width. Alternatively put, the order formation in our model involves the increase in entropy followed by its decrease, that is entropy fluctuation or oscillation. In comparison, BP is mainly driven by the macroscopic motion due to the movement of the PDF peak with much less prominent appearance of a geodesic solution.

The time scale $\tau$ of the information change and the information length $\mathcal{L}_{\infty}=\mathcal{L}(t \rightarrow \infty)$ also behave very differently in FPs and BPs. In particular, the FP supports an interesting geodesic solution with constant $\tau=1 /(\sqrt{2} \gamma)$, independent of the strength of the stochastic noise $D$, along which the information flows at the constant rate $\tau^{-1}=\sqrt{2} \gamma$. Notably, this geodesic involves the broadening followed by a narrowing of a PDF (that is, the fluctuation or oscillation in the PDF width) and was traced back to self-regulation between positive and negative feedback and the subtle energy balance between the linear growth and nonlinear damping in our model. Comparing with the linear OU process where a geodesic was possible only by explicitly time-dependent model parameters, these results show that a nonlinear interaction involved in disorderto-order transition promotes a geodesic solution. Alternatively, the self-regulation between the positive feedback $(\gamma x)$ and the negative feedback $\left(-\mu x^{3}\right)$ maintains the system closer to the geodesic, minimizing the information change. In a biological context, this minimal geodesic path can be understood in terms of "fitness" in the growth phase (e.g., gene expression). We suggest that the predator-prey type self-regulation between the positive feedback and the negative feedback in the disorder-to-order transition maintains the system closer to the geodesic, minimizing the information change. That is, selfregulation with a nonlinear interaction facilitates a geodesic.

The total information length $\mathcal{L}_{\infty}$ between initial and final states is consequently much larger in BPs than in FPs. Specifically, it increases linearly with the deviation $\gamma$ of a control parameter from the critical state in BPs while increasing logarithmically with $\gamma$ in FPs. As $\mathcal{L}_{\infty}$ due to the macroscopic motion is a more useful form of the energy that can be extracted, a larger $\mathcal{L}_{\infty}$ in BPs than in FPs suggests the possibility of extracting a net useful energy from a pair of FP and BP processes, e.g., in a cyclic order-disorder transition, often observed in nature. On the other hand, from the perspective of the fitness of a system discussed above and in Sec. I, a smaller $\mathcal{L}$ in the FP can be considered to be advantageous for the system when adjusting to a changing environment takes time and/or is costly. Furthermore, we showed that $\mathcal{L}$ has the great capability of capturing different physical processes - diffusion or advection involved in FPs or BPs-by geometry. In particular, $\mathcal{L}_{\infty} \propto|\ln D|$ and $D^{-1 / 2}$ in FPs and BPs, respectively, reveal drastically different roles of diffusion $D$ in the FP or BP transition.

Finally, we note that order-to-disorder transition always occurred faster than disorder-to-order transition, as often observed in self-organizing systems. These results suggest that a cyclic order-disorder transition in our toy model could serve as a useful simple model to capture dynamic equilibrium in self-organizing systems which maintain a quasiequilibrium, repeating the formation and disappearance of a coherent structure. Building upon the understanding of our OD model within which a detailed mathematical analysis was possible, it would be of great interest to extend this model to a more realistic case (e.g., 1D or 2D models, a system of coupled equations, etc.).

\section{APPENDIX A: RELATION BETWEEN $\mathcal{L}$ AND RELATIVE ENTROPY}

We first show the relation between $\tau(t)$ in Eq. (2) and the second derivative of the relative entropy (or KullbackLeibler divergence) $D\left(p_{1}, p_{2}\right)=\int d x p_{2} \ln \left(p_{2} / p_{1}\right)$, where $p_{1}=p\left(x, t_{1}\right)$ and $p_{2}=p\left(x, t_{2}\right)$, as follows:

$$
\begin{gathered}
\frac{\partial}{\partial t_{1}} D\left(p_{1}, p_{2}\right)=-\int d x p_{2} \frac{\partial_{t_{1}} p_{1}}{p_{1}}, \\
\frac{\partial^{2}}{\partial t_{1}^{2}} D\left(p_{1}, p_{2}\right)=\int d x p_{2}\left[\frac{\left(\partial_{t_{1}} p_{1}\right)^{2}}{p_{1}^{2}}-\frac{\partial_{t_{1}}^{2} p_{1}}{p_{1}}\right], \\
\frac{\partial}{\partial t_{2}} D\left(p_{1}, p_{2}\right)=\int d x\left[\partial_{t_{2}} p_{2}+\partial_{t_{2}} p_{2}\left(\ln p_{2}-\ln p_{1}\right)\right],
\end{gathered}
$$




$$
\begin{aligned}
\frac{\partial^{2}}{\partial t_{2}^{2}} D\left(p_{1}, p_{2}\right)= & \int d x\left[\partial_{t_{2}}^{2} p_{2}+\frac{\left(\partial_{t_{2}} p_{2}\right)^{2}}{p_{2}}\right. \\
& \left.+\partial_{t_{2}}^{2} p_{2}\left(\ln p_{2}-\ln p_{1}\right)\right] .
\end{aligned}
$$

By taking the limit where $t_{2} \rightarrow t_{1}=t\left(p_{2} \rightarrow p_{1}=p\right)$ and by using the total probability conservation (e.g., $\int d x \partial_{t} p=0$ ), Eqs. (A1) and (A3) above lead to

$$
\lim _{t_{2} \rightarrow t_{1}=t} \frac{\partial}{\partial t_{1}} D\left(p_{1}, p_{2}\right)=\lim _{t_{2} \rightarrow t_{1}=t} \frac{\partial}{\partial t_{2}} D\left(p_{1}, p_{2}\right)=\int d x \partial_{t} p=0,
$$

while Eqs. (A2) and (A4) give

$$
\lim _{t_{2} \rightarrow t_{1}=t} \frac{\partial^{2}}{\partial t_{1}^{2}} D\left(p_{1}, p_{2}\right)=\lim _{t_{2} \rightarrow t_{1}=t} \frac{\partial^{2}}{\partial t_{2}^{2}} D\left(p_{1}, p_{2}\right)=\int d x \frac{\left(\partial_{t} p\right)^{2}}{p} \text {. }
$$

See also [38] for a similar derivation.

To link this to information length $\mathcal{L}$, we then express $D\left(p_{1}, p_{2}\right)$ for small $d t=t_{2}-t_{1}$ as

$$
D\left(p_{1}, p_{2}\right)=\frac{1}{2}\left[\int d x \frac{\left(\partial_{t_{1}} p\left(x, t_{1}\right)\right)^{2}}{p}\right](d t)^{2}+O\left[(d t)^{3}\right],
$$

where $O\left[(d t)^{3}\right]$ is a higher order term in $d t$. We define the infinitesimal distance (information length) $d l\left(t_{1}\right)$ between $t_{1}$ and $t_{1}+d t$ by

$$
d l\left(t_{1}\right)=\sqrt{D\left(p_{1}, p_{2}\right)}=\frac{1}{\sqrt{2}} \sqrt{\int d x \frac{\left(\partial_{t} p\right)^{2}}{p}} d t+O\left[(d t)^{3 / 2}\right] .
$$

The total change in information between time $t=0$ and $t$ is then obtained by summing over $d t\left(t_{1}\right)$ and taking the limit $d t \rightarrow 0$ as

$$
\begin{aligned}
\mathcal{L}(t)= & \lim _{d t \rightarrow 0}[d l(0)+d l(d t)+d l(2 d t) \\
& +d l(3 d t)+\cdots d l(t-d t)] \\
= & \lim _{d t \rightarrow 0}[\sqrt{D(p(x, 0), p(x, d t))}+\sqrt{D(p(x, d t), p(x, 2 d t))} \\
& +\cdots \sqrt{D(p(x, t-d t), p(x, t))}] \\
\propto & \int_{0}^{t} d t_{1} \sqrt{\int d x \frac{\left(\partial_{t_{1}} p\right)^{2}}{p}} .
\end{aligned}
$$

\section{APPENDIX B: DERIVATION OF EQS. (7)-(10)}

To find the equilibrium PDFs $p_{F}(x)$ and $p_{B}(x)$, we look for the stationary solution of the Fokker-Planck equation, Eq. (6);

$$
0=\frac{\partial}{\partial t} p(x, t)=\frac{\partial}{\partial x}\left[-F(x)+D \frac{\partial}{\partial x}\right] p(x, t),
$$

and find that

$$
p(x, t \rightarrow \infty) \propto \exp \left(-\frac{1}{D} \int^{x} d x_{1} F\left(x_{1}\right)\right) .
$$

For the FP, $F(x)=-\gamma x+\mu x^{3}$, and thus

$$
\begin{aligned}
p(x, t \rightarrow \infty) & =p_{F} \propto \exp \left(-\frac{1}{4 D}\left[2 \gamma x^{2}-\mu x^{4}\right]\right) \\
& \propto \exp \left(-\frac{\mu}{4 D}\left[x^{2}-\frac{\gamma}{\mu}\right]^{2}\right),
\end{aligned}
$$

For the BP, $F(x)=\gamma x+\mu x^{3}$, and thus

$$
\begin{aligned}
p(x, t \rightarrow \infty) & \left.=p_{F} \propto \exp \left(-\frac{1}{4 D}\left[2 \gamma x^{2}+\mu x^{4}\right)\right]\right) \\
& \propto \exp \left(-\frac{\mu}{4 D}\left[x^{2}+\frac{\gamma}{\mu}\right]^{2}\right) .
\end{aligned}
$$

For sufficiently small $D$, we can approximate $p_{F}(x)$ by expanding it around the two peaks $x= \pm \sqrt{\frac{\gamma}{\mu}}$. Specifically, around $x=\sqrt{\frac{\gamma}{\mu}}$,

$$
\left[x^{2}-\frac{\gamma}{\mu}\right]^{2}=\left[x-\sqrt{\frac{\gamma}{\mu}}\right]^{2}\left[x+\sqrt{\frac{\gamma}{\mu}}\right]^{2} \sim 4 \frac{\gamma}{\mu}\left[x-\sqrt{\frac{\gamma}{\mu}}\right]^{2} .
$$

Similarly, around $x=-\sqrt{\frac{\gamma}{\mu}}$,

$\left[x^{2}-\frac{\gamma}{\mu}\right]^{2}=\left[x-\sqrt{\frac{\gamma}{\mu}}\right]^{2}\left[x+\sqrt{\frac{\gamma}{\mu}}\right]^{2} \sim 4 \frac{\gamma}{\mu}\left[x+\sqrt{\frac{\gamma}{\mu}}\right]^{2}$.

Thus, these results enable us to approximate $p_{F}(x)$ by the double Gaussian given by Eq. (9) in the text. For the BP, for small $D$, the PDF has a narrow peak around $x=0$. Thus, by neglecting $x^{4}$ in Eq. (8) in comparison with $x^{2}$, we obtain Eq. (10).

\section{APPENDIX C: DERIVATION OF EQ. (23)}

In order to compute the second moment, we utilize $p(x, t) d x=p(y, t) d y$ :

$$
\begin{aligned}
\left\langle x^{2}\right\rangle & =\int_{-\infty}^{\infty} d x p(x, t) x^{2}=\int_{-\infty}^{\infty} d y p(y, t) x^{2} \\
& =\int_{-\infty}^{\infty} d y x^{2} \sqrt{\frac{\beta}{\pi}} e^{-\beta y^{2}} \\
& =2 \int_{0}^{\infty} d y \frac{y^{2}}{1+\alpha y^{2}} \sqrt{\frac{\beta}{\pi}} e^{-\beta y^{2}} \\
& =\frac{2}{\alpha}\left[\frac{1}{2}-\sqrt{\frac{\beta}{\pi}} I\right],
\end{aligned}
$$

where the integral $I \equiv \int_{0}^{\infty} \frac{1}{1+\alpha y^{2}} e^{-\beta y^{2}}$. In Eq. $(\mathrm{C} 1), \operatorname{Erfc}(Q)=$ $\int_{Q}^{\infty} d y e^{-y^{2}}=\frac{\sqrt{\pi}}{2} \operatorname{erfc}(Q)$ where $\operatorname{erfc}(Q)=\frac{2}{\sqrt{\pi}} \int_{Q}^{\infty} d y e^{-y^{2}}$ is the complementary error function. In order to compute $I \equiv$ $\int_{0}^{\infty} \frac{1}{1+\alpha y^{2}} e^{-\beta y^{2}}$, we differentiate $I$ with respect to $\beta$ and obtain 
the following differential equation:

$$
\begin{aligned}
\partial_{\beta} I & =-\int_{0}^{\infty} d y \frac{y^{2}}{1+\alpha y^{2}} e^{-\beta y^{2}} \\
& =-\frac{1}{\alpha}\left[\frac{1}{2} \sqrt{\frac{\beta}{\pi}}-\int_{0}^{\infty} \frac{1}{1+\alpha y^{2}} e^{-\beta y^{2}}\right] \\
& =-\frac{1}{2 \alpha} \sqrt{\frac{\beta}{\pi}}+\frac{1}{\alpha} I .
\end{aligned}
$$

Thus,

$$
\partial_{\beta} I-\frac{1}{\alpha} I=-\frac{1}{2 \alpha} \sqrt{\frac{\beta}{\pi}} .
$$

We solve Eq. (C3) as

$$
I(\beta)=I\left(\beta_{0}\right) e^{\beta / \alpha}-\sqrt{\frac{\pi}{2 \alpha}} e^{\beta / \alpha} J,
$$

where $\beta_{0}=0$ and

$$
\begin{aligned}
I\left(\beta_{0}\right) & =\int_{0}^{\infty} \frac{1}{1+\alpha y^{2}}=\frac{\pi}{2 \sqrt{\alpha}}, \\
J & =\int_{\beta_{0}}^{\beta} \frac{d \beta_{1}}{\sqrt{\beta_{1}}} e^{-\beta_{1} / \alpha} .
\end{aligned}
$$

$J$ in Eq. (C5) is calculated as follows:

$$
\begin{aligned}
J & =2 \int_{0}^{\sqrt{\beta}} e^{-z^{2} / \alpha} \\
& =2 \sqrt{\alpha} \int_{0}^{\sqrt{\beta / \alpha}} d p e^{-p^{2}} \\
& =\sqrt{\alpha \pi}-2 \sqrt{\alpha} \operatorname{Erfc}\left(\sqrt{\frac{\beta}{\alpha}}\right) .
\end{aligned}
$$

By substituting Eq. (C6) in Eq. (C4), we obtain

$$
I=\sqrt{\frac{\pi}{\alpha}} e^{\frac{\beta}{\alpha}} \operatorname{Erfc}\left(\sqrt{\frac{\beta}{\alpha}}\right) .
$$

By inserting Eq. (C7) in Eq. (C1), we obtain

$$
\left\langle x^{2}\right\rangle=\frac{2}{\alpha}\left[\frac{1}{2}-\sqrt{\frac{\beta}{\alpha}} e^{\frac{\beta}{\alpha}} \operatorname{Erfc}\left(\sqrt{\frac{\beta}{\alpha}}\right)\right],
$$

which is Eq. (23) in Sec. III.

\section{APPENDIX D: DERIVATION OF EQ. (24)}

In order to compute the fourth moment, we again utilize $p(x, t) d x=p(y, t) d y$ :

$$
\begin{aligned}
\left\langle x^{4}\right\rangle & =\int_{-\infty}^{\infty} d y p(y, t) x^{4}=\int_{-\infty}^{\infty} d y x^{4} \sqrt{\frac{\beta}{\pi}} e^{-\beta y^{2}} \\
& =2 \int_{0}^{\infty} d y \frac{y^{4}}{\left(1+\alpha y^{2}\right)^{2}} \sqrt{\frac{\beta}{\pi}} e^{-\beta y^{2}} \\
& =\frac{2}{\alpha^{2}}\left[\frac{1}{2}+\sqrt{\frac{\beta}{\pi}}(-2 I+K)\right],
\end{aligned}
$$

where $\quad K \equiv \int_{0}^{\infty} \frac{1}{\left(1+\alpha y^{2}\right)^{2}} e^{-\beta y^{2}}$. To evaluate $K \equiv$ $\int_{0}^{\infty} \frac{1}{\left(1+\alpha y^{2}\right)^{2}} e^{-\beta y^{2}}$, we rewrite $I$ by using $\alpha=\gamma / \mu$ as

$$
I=\gamma \int_{0}^{\infty} \frac{1}{\gamma+\mu y^{2}} e^{-\beta y^{2}}
$$

and consider

$$
\begin{aligned}
\partial_{\gamma}\left(\frac{I}{\gamma}\right) & =\partial_{\gamma} \int_{0}^{\infty} \frac{1}{\gamma+\mu y^{2}} e^{-\beta y^{2}} \\
& =-\frac{1}{\gamma^{2}} \int_{0}^{\infty} \frac{1}{\left(1+\alpha y^{2}\right)^{2}} e^{-\beta y^{2}} .
\end{aligned}
$$

Equation (D3) gives

$$
K=-\gamma^{2} \partial_{\gamma}\left(\frac{I}{\gamma}\right)
$$

We rewrite $I / \gamma$ by using $\alpha=\gamma / \mu$,

$$
\begin{aligned}
\frac{I}{\gamma} & =\sqrt{\frac{\pi}{\mu \gamma}} e^{\beta \gamma / \mu} \operatorname{Erfc}\left(\sqrt{\frac{\beta \gamma}{\mu}}\right) \\
& =\sqrt{\frac{\pi}{\mu \gamma}} e^{\beta \gamma / \mu} \int_{\sqrt{\beta \gamma / \mu}}^{\infty} d p e^{-p^{2}} .
\end{aligned}
$$

We then obtain from Eq. (D5)

$$
\partial_{\gamma}\left(\frac{I}{\gamma}\right)=\left(-\frac{1}{2 \gamma}+\frac{\beta}{\mu}\right) \frac{I}{\gamma}-\frac{1}{2 \gamma \mu} \sqrt{\beta \pi} .
$$

Using Eq. (D6) in (D1) gives us

$$
\left\langle x^{4}\right\rangle=\frac{2}{\alpha^{2}}\left[\frac{1}{2}-\left(\frac{3}{2}+\frac{\beta}{\alpha}\right) \sqrt{\frac{\beta}{\alpha}} e^{\beta / \alpha} \operatorname{Erfc}\left(\sqrt{\frac{\beta}{\alpha}}\right)+\frac{\beta}{2 \alpha}\right],
$$

which is Eq. (24) in the text.

\section{APPENDIX E: PROPERTIES OF THE SUM OF TWO GAUSSIAN PDFS}

In this Appendix, we show that the information length for double Gaussian PDFs which are well separated is approximately the same as that for a single Gaussian PDF. To this end, we let

$$
\begin{aligned}
p & =p_{1}+p_{2}=N(t)\left[\tilde{p}_{1}+\tilde{p}_{2}\right], \\
N(t) & =\frac{\sqrt{\beta(t)}}{2 \sqrt{\pi}} \\
\tilde{p}_{1} & =e^{-\beta(t)\left(x+x_{0}\right)^{2}}=e^{-\beta(t) x_{1}^{2}} \\
\tilde{p}_{2} & =e^{-\beta(t)\left(x-x_{0}\right)^{2}}=e^{-\beta(t) x_{2}^{2}}
\end{aligned}
$$

Here, $N$ is the normalization constant [e.g., $N^{-1}=\int d x\left(\tilde{p}_{1}+\right.$ $\left.\tilde{p}_{2}\right)$ ] and $x_{1}=x+x_{0}$ and $x_{2}=x-x_{0}$.

To show Eq. (44), we assume $x_{0}$ is constant given by the peak location $x_{0}=\sqrt{\frac{\gamma}{\mu}}$ in $x>0$ while $\beta=\beta(t)$ depending 
on time. Then, we can show

$$
\begin{aligned}
\frac{1}{p(x, t)}\left[\frac{\partial p(x, t)}{\partial t}\right]^{2}= & \frac{\dot{N}^{2}}{N}\left(\tilde{p}_{1}+\tilde{p}_{2}\right)+2 \dot{N}\left(\dot{\tilde{p}}_{1}+\dot{\tilde{p}}_{2}\right) \\
& +N \frac{\left(\dot{\tilde{p}}_{1}+\dot{\tilde{p}}_{2}\right)^{2}}{\tilde{p}_{1}+\tilde{p}_{2}} .
\end{aligned}
$$

Now, we compute the various quantities in Eq. (E2) as follows:

$$
\begin{aligned}
\dot{\tilde{p}}_{1} & =-\dot{\beta} x_{1}^{2} \tilde{p}_{1}=\dot{\beta} \partial_{\beta} \tilde{p}_{1}, \\
\left(\dot{\tilde{p}}_{1}\right)^{2} & =\dot{\beta}^{2} \tilde{p}_{1} \partial_{\beta \beta} \tilde{p}_{1} .
\end{aligned}
$$

Similarly,

$$
\begin{aligned}
\dot{\tilde{p}}_{2} & =-\dot{\beta} x_{2}^{2} \tilde{p}_{2}=\dot{\beta} \partial_{\beta} \tilde{p}_{2}, \\
\left(\dot{\tilde{p}}_{2}\right)^{2} & =\dot{\beta}^{2} \tilde{p}_{2} \partial_{\beta \beta} \tilde{p}_{2} .
\end{aligned}
$$

Thus, by using Eqs. (E3) and (E4), we calculate the last term in Eq. (E2) as follows:

$$
\begin{aligned}
\left(\dot{\tilde{p}}_{1}+\dot{\tilde{p}}_{2}\right)^{2} & =\dot{\beta}^{2}\left[\tilde{p}_{1} \partial_{\beta \beta} \tilde{p}_{1}+\tilde{p}_{2} \partial_{\beta \beta} \tilde{p}_{2}+2 \partial_{\beta} \tilde{p}_{1} \partial_{\beta} \tilde{p}_{2}\right] \\
& =\dot{\beta}^{2}\left[\left(\tilde{p}_{1}+\tilde{p}_{2}\right) \partial_{\beta \beta} \tilde{p}_{1}+\left(\tilde{p}_{1}+\tilde{p}_{2}\right) \partial_{\beta \beta} \tilde{p}_{2}+G_{1}\right] \\
& =\dot{\beta}^{2}\left[\left(\tilde{p}_{1}+\tilde{p}_{2}\right) \partial_{\beta \beta}\left(\tilde{p}_{1}+\tilde{p}_{2}\right)+G_{2}\right],
\end{aligned}
$$

where $G_{1}$ and $G_{2}$ are terms involving the product of $\tilde{p}_{1}$ and $\tilde{p}_{2}$. For the PDF peaks that are well separated and thus independent, there is no overlap between $\tilde{p}_{1}$ and $\tilde{p}_{2}$ in $x$, leading to $\int d x \tilde{p}_{1}(x) \tilde{p}_{2}(x)=0$. That is, in this case, $\int d x G_{1}=\int d x G_{2}=0$. Thus, these terms $G_{1}$ and $G_{2}$ do not contribute to Eq. (2). By using these results in Eq. (2), we obtain

$$
\int d x \frac{1}{p(x, t)}\left[\frac{\partial p(x, t)}{\partial t}\right]^{2}=\frac{\dot{N}^{2}}{N^{2}}+2 \dot{\beta} \dot{N} \partial_{\beta} \frac{1}{N}+N \dot{\beta}^{2} \partial_{\beta \beta} \frac{1}{N} .
$$

By using $N=\frac{1}{2} \sqrt{\frac{\beta}{\pi}}$, we simplify Eq. (E8) as

$$
\int d x \frac{1}{p(x, t)}\left[\frac{\partial p(x, t)}{\partial t}\right]^{2}=\frac{\dot{\beta}^{2}}{2 \beta^{2}}=\frac{\dot{\sigma}^{2}}{2 \sigma^{2}} .
$$

Thus, Eq. (E9) is the same as Eq. (39) in the limit $z=0$. We note that Eq. (44) is obtained by the time integral of Eq. (E9).

Next to show Eq. (47), we need to consider the case where $\beta$ is constant in Eq. (E1) while $x_{0}=x_{0}(t)$ depends on time. In this case, we have

$$
\begin{aligned}
\left(\dot{\tilde{p}}_{1}+\dot{\tilde{p}}_{2}\right)^{2}= & 4 \beta^{2} \dot{x}_{0}^{2} N^{2}\left[x^{2}\left(\tilde{p}_{1}+\tilde{p}_{2}\right)^{2}+2 x x_{0}\left(\tilde{p}_{1}^{2}-\tilde{p}_{2}^{2}\right)\right. \\
& \left.+x_{0}^{2}\left(\tilde{p}_{1}-\tilde{p}_{2}\right)^{2}\right] \\
= & 4 \beta^{2} \dot{x}_{0}^{2} N^{2}\left[x^{2}\left(\tilde{p}_{1}+\tilde{p}_{2}\right)^{2}+2 x x_{0}\left(\tilde{p}_{1}^{2}-\tilde{p}_{2}^{2}\right)\right. \\
& \left.+x_{0}^{2}\left(\tilde{p}_{1}+\tilde{p}_{2}\right)^{2}+G_{3}\right],
\end{aligned}
$$

where $G_{3}$ is a function depending on the product of $\tilde{p}_{1}$ and $\tilde{p}_{2}$, which vanishes upon integration over $x$ when $\tilde{p}_{1}$ and $\tilde{p}_{2}$ are well separated with negligible overlap. In this case,

$$
\begin{aligned}
& \int d x \frac{1}{p(x, t)}\left[\frac{\partial p(x, t)}{\partial t}\right]^{2} \\
& =4 \beta^{2} \dot{x}_{0}^{2} N \int d x\left[\left(x+x_{0}\right)^{2} \tilde{p}_{1}+\left(x-x_{0}\right)^{2} \tilde{p}_{2}\right] \\
& =-4 \beta^{2} \dot{x}_{0}^{2} N \partial_{\beta} \int d x\left(\tilde{p}_{1}+\tilde{p}_{2}\right) \\
& =-4 \beta^{2} \dot{x}^{2} N \partial_{\beta} \frac{1}{N} \\
& =2 \beta \dot{x}_{0}^{2}
\end{aligned}
$$

where we used $N=\frac{1}{2} \sqrt{\frac{\beta}{\pi}}$ and thus $\partial_{\beta} \frac{1}{N}=-\frac{1}{2 \beta N}$. Equation (E11) is the same as Eq. (39) in the opposite limit where $z=x_{0}$ and $\dot{\beta}=0$.

\section{APPENDIX F: TWO INDEPENDENT GAUSSIAN PDFS}

It is interesting to consider what would happen in the case of the broken ergodicity such that the PDFs in $x>0$ and $x<0$ are independent. To this end, we recast Eq. (10) as

$$
\begin{aligned}
& p_{F}=\frac{1}{2}\left[p_{1}+p_{2}\right], \\
& p_{1}=\frac{\sqrt{\beta_{F}}}{\sqrt{\pi}} e^{-(\gamma / D)(x+\sqrt{\gamma / \mu})^{2}}, \\
& p_{2}=\frac{\sqrt{\beta_{F}}}{\sqrt{\pi}} e^{-(\gamma / D)(x-\sqrt{\gamma / \mu})^{2}},
\end{aligned}
$$

where $\beta_{F}=\gamma / D$, and compute the configurational entropy [51] by taking into account the probability of $1 / 2$ of $x$ to be in $x>0$ or $x<0$ as follows:

$$
\begin{aligned}
S_{F C} & =-\frac{1}{2} \int_{-\infty}^{\infty} d x\left[p_{1} \ln p_{1}+p_{2} \ln p_{2}\right] \\
& =-\int_{-\infty}^{\infty} d x p_{1} \ln p_{1}=-\frac{1}{2}\left[1+\ln \frac{\pi D}{\gamma}\right],
\end{aligned}
$$

where we used $\int_{-\infty}^{\infty} d x p_{1} \ln p_{1}=\int_{-\infty}^{\infty} d x p_{2} \ln p_{2}$ due to symmetry under $x \rightarrow-x$. Interestingly, Eqs. (51) and (F2) differ by $\ln 2$ due to the broken ergodicity (i.e., due to the reduction of a phase space by half). This difference of $\ln 2$ is however negligible for small $D$ and $S_{F} \sim S_{F c} \sim-\frac{1}{2} \ln \frac{\pi D}{\gamma}$. On the other hand, $S_{F c}$ in Eq. (F2) taking into account the breakdown of ergodicity is smaller than $S_{B}$ by $\frac{1}{2} \ln 2$ as the ergodic phase region is reduced to $x>0$ or $x<0$. Again, this difference is very small compared to the contribution from $D$.
[1] T. W. B. Kibble, Phys. Rep. 67, 183 (1980).

[2] Y. Nagashima and Y. Nambu, Elementary Particle Physics: Quantum Field Theory and Particles (Wiley-VCH, Weinheim, 2010).
[3] G. Mazenko, Physica A 204, 437 (1994).

[4] G. Longo and M Montévil, Prog. Biophys. Mol. Biol. 106, 340 (2011)

[5] T. Bossomaier, L. Barnett, and M. Harré, Complex Adapt. Syst. Model. 1, 9 (2013). 
[6] H. Haken, Information and Self-organization: A Macroscopic Approach to Complex Systems (Springer, Berlin, 2006).

[7] E. Kim and P. H. Diamond, Phys. Rev. Lett. 90, 185006 (2003).

[8] E. Kim, Phys. Rev. Lett. 96, 084504 (2006).

[9] K. Srinivasan and W. R. Young, J. Atmos. Sci. 69, 1633 (2012).

[10] K. M. Sayanagi, A. P. Showman, and T. E. Dowling, J. Atmos. Sci. 65, 12 (2008).

[11] E. Kim, H.-L. Liu, and J. Anderson, Phys. Plasmas 16, 052304 (2009).

[12] A. P. L. Newton, E. Kim, and H.-L. Liu, Phys. Plasmas 20, 092306 (2013).

[13] M. Tsuchiya, A. Giuliani, M. Hashimoto, J. Erenpreisa, and K. Yoshikawa, PLoS One 10, e0128565 (2015).

[14] C. Tang and P. Bak, J. Stat. Phys. 51, 797 (1988).

[15] H. J. Jensen, Self-organized Criticality: Emergent Complex Behavior in Physical and Biological Systems (Cambridge University Press, Cambridge, England, 1998).

[16] G. Pruessner, Self-organised Criticality (Cambridge University Press, Cambridge, England, 2012).

[17] S. Fauve and F. Heslot, Phys. Lett. A 97, 5 (1983).

[18] M. L. Ferguson, D. Le Coq, M. Jules, S. Aymerich, O. Radulescue, N. Declerck, and C. A. Royer, Proc. Natl. Acad. Sci. USA 109, 155 (2012).

[19] D. Angeli, J. E. Ferrell, and E. D. Sontag, Proc. Natl. Acad. Sci. USA 101, 1822 (2004).

[20] D. Holcman and M. Tsodyks, PLoS Comp. Biol. 2, e23 (2006).

[21] J. F. Mejias, H. J. Kappen, and J. J. Torres, PLoS One 5, e13651 (2010).

[22] J. Hidalgo, L. F. Seoane, J. M. Cortés, and M. A. Munoz, PloS One 7, e40710 (2012)

[23] S. Tyagi, Mol. Syst. Biol. 11, 805 (2015).

[24] S. di Santo, R. Burioni, A. Vezzani, and M. A. Muñoz, Phys. Rev. Lett. 116, 240601 (2016).

[25] S. B. Nicholson and E. Kim, Phys. Lett. A379, 83 (2015).

[26] S. B. Nicholson and E. Kim, Entropy 18, 258 (2016).

[27] J. Heseltine and E. Kim, J. Phys. A 49, 175002 (2016).
[28] E. Kim, U. J. Lee, J. Heseltine, and R. Hollerbach, Phys. Rev. E 93, 062127 (2016).

[29] E. Kim and R. Hollerbach, Phys. Rev. E 95, 022137 (2017).

[30] A. L. Gibbs and F. E. Su, Int. Stat. Rev. 70, 419 (2002).

[31] W. K. Wootters, Phys. Rev. D 23, 357 (1981).

[32] G. Ruppeiner, Phys. Rev. A 20, 1608 (1979).

[33] F. Schlögl, Z. Phys. B: Condens. Matter 59, 449 (1985).

[34] E. H. Feng and G. E. Crooks, Phys. Rev. E. 79, 012104 (2009).

[35] S. L. Braunstein and C. M. Caves, Phys. Rev. Lett. 72, 3439 (1994).

[36] H. Strobel, W. Muessel, D. Linnemann, T. Zibold, D. B. Hume, L. Pezzé, A. Smerzi, and M. K. Oberthaler, Science 345, 424 (2014).

[37] J. Nulton, P. Salamon, B. Andresen, and Q. Anmin, J Chem. Phys. 83, 334 (1985).

[38] G. E. Crooks, Phys. Rev. Lett. 99, 100602 (2007).

[39] D. A. Sivak and G. E. Crooks, Phys. Rev. Lett. 108, 190602 (2012).

[40] P. Salamon, J. D. Nulton, G. Siragusa, A. Limon, D. Bedeaux, and S. Kjelstrup, J. Non-Equilib. Thermodyn. 27, 45 (2002).

[41] F. Klebaner, Introduction to Stochastic Calculus with Applications (Imperial College Press, London, 2012).

[42] H. Risken, The Fokker-Planck Equation: Methods of Solutions and Applications (Springer, Berlin, 1996).

[43] J. K. Bhattacharjee, P. Meakin, and D. J. Scalapino, Phys. Rev. A 30, 1026 (1984).

[44] E. Kim and R. Hollerbach, Phys. Rev. E 94, 052118 (2016).

[45] M. Suzuki, Prog. Theor. Phys. 56, 77, (1976).

[46] B. Caroli, C. Caroli, and B. Roulet, J. Stat. Phys. 21, 415 (1979).

[47] R. Kubo, K. Matsuo, and K. Kitahara, J. Stat. Phys. 9, 51 (1973).

[48] M. Suzuki, J. Stat. Phys. 16, 477 (1977).

[49] M. Suzuki, Phys. Lett. A 67, 339 (1978).

[50] J. V. Michalowicz, J. M. Nichols, and F. Bucholtz, Entropy 10, 200 (2008).

[51] J. C. Mauro, R. J. Loucks, and S. Sen, J. Chem. Phys. 133, 164503 (2010). 